ARTICLE

\title{
Dietary serine-microbiota interaction enhances chemotherapeutic toxicity without altering drug conversion
}

\author{
Wenfan Ke (10 1,7, James A. Saba (1) 1,7, Cong-Hui Yao (1) 2, Michael A. Hilzendeger (10 1, Anna Drangowska-Way?, \\ Chintan Joshi ${ }^{3,4}$, Vinod K. Mony ${ }^{1}$, Shawna B. Benjamin'1, Sisi Zhang ${ }^{2}$, Jason Locasale ${ }^{5}$, Gary J. Patti ${ }^{2}$, \\ Nathan Lewis ${ }^{3,4 凶}$ \& Eyleen J. O'Rourke (iD ${ }^{1,6 \times}$
}

The gut microbiota metabolizes drugs and alters their efficacy and toxicity. Diet alters drugs, the metabolism of the microbiota, and the host. However, whether diet-triggered metabolic changes in the microbiota can alter drug responses in the host has been largely unexplored. Here we show that dietary thymidine and serine enhance 5-fluoro 2'deoxyuridine (FUdR) toxicity in $C$. elegans through different microbial mechanisms. Thymidine promotes microbial conversion of the prodrug FUdR into toxic 5-fluorouridine-5'-monophosphate (FUMP), leading to enhanced host death associated with mitochondrial RNA and DNA depletion, and lethal activation of autophagy. By contrast, serine does not alter FUdR metabolism. Instead, serine alters E. coli's $1 C$-metabolism, reduces the provision of nucleotides to the host, and exacerbates DNA toxicity and host death without mitochondrial RNA or DNA depletion; moreover, autophagy promotes survival in this condition. This work implies that diet-microbe interactions can alter the host response to drugs without altering the drug or the host.

\footnotetext{
${ }^{1}$ Department of Biology, College of Arts and Sciences, University of Virginia, Charlottesville, VA, USA. ${ }^{2}$ Department of Chemistry, Washington University, St. Louis, MO, USA. ${ }^{3}$ Department of Pediatrics, University of California, San Diego, La Jolla, CA, USA. ${ }^{4}$ Department of Bioengineering, University of California, San Diego, La Jolla, CA, USA. ${ }^{5}$ Department of Pharmacology and Cancer Biology, Duke University, Durham, NC, USA. ${ }^{6}$ Department of Cell Biology, School of

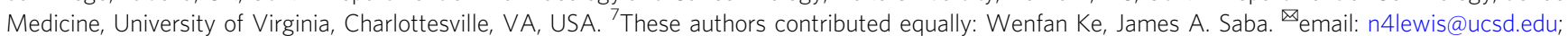
ejo8b@virginia.edu
} 
$\longrightarrow$ by lassically, diet has been thought to modulate drug efficacy and toxicity through altering the physiology of the host or by directly interfering with the pharmacodynamics of the drug $^{1,2}$. However, emerging evidence shows that diet can also modulate drug efficacy and toxicity through modifying the composition or physiology of the microbiota, or the interaction between the microbiota and the host ${ }^{3}$. In this study, we utilize a tractable model system to uncover and mechanistically dissect a four-way interaction between the amino acid serine (diet), the chemotherapeutic $5^{\prime}$-fluorodeoxyuridine (drug), the bacterium $E$. coli (microbiota), and the roundworm $C$. elegans (host).

Fluoropyrimidines are commonly used chemotherapeutics, especially for cancers of the GI tract ${ }^{4}$. The most accepted mechanism of action of fluoropyrimidines is inhibition of thymidylate synthase (TS). TS catalyzes the methylation of $2^{\prime}$ deoxyuridine- $5^{\prime}$-monophosphate (dUMP) in position 5 of the uracil ring to produce $2^{\prime}$-deoxythymidine- $5^{\prime}$-monophosphate (dTMP). TS uses the 1-carbon (1C) metabolite 5,10-methylenetetrahydrofolate $(5,10-\mathrm{mTHF})$ as the indispensable methyl-group donor. TS is critical for cell survival and replication as it is the sole biosynthetic source of dTMP, which is essential for DNA synthesis. When cells are treated in vitro with the fluoropyrimidine 5 -fluorodeoxyuridine (FUdR), they convert FUdR into $5^{\prime}$-fluorodeoxyuridine monophosphate (FdUMP). FdUMP is structurally similar to dUMP, except that it has a fluorine atom in position 5 of the uracil ring. As a consequence, FdUMP forms a stable complex with 5,10-mTHF and TS, preventing the de novo synthesis of dTMP. 5,10-mTHF is essential for dTMP synthesis and for the FdUMP-mediated inhibition of TS 5 . 1C-loaded folates are not known to transfer across membranes; thus, 5,10-mTHF must be locally generated ${ }^{5}$. 5,10-mTHF can be made from the amino acids serine and glycine. Glycine can be degraded via the glycine cleavage system (GCS) to generate $\mathrm{NH}_{3}, \mathrm{CO}_{2}$, and a methyl group that is incorporated into 5,10-mTHF. Separately, the reaction that converts serine to glycine also donates a $1 \mathrm{C}$ group to THF to form 5,10-mTHF, which is then available to participate in the methyl transfer reaction that converts dUMP into dTMP. Indeed, $1 \mathrm{C}$ units derived from radiolabeled serine are incorporated into nucleotides ${ }^{6}$. Importantly, the levels of 5,10mTHF are known to limit the efficacy of fluoropyrimidines ${ }^{4,7}$.

Several 1C-metabolites are obtained directly or indirectly from the diet, and the therapeutic value of their dietary supplementation is widely exploited ${ }^{5}$. Serving as a substrate for the synthesis of 5,10-mTHF, the 1C-metabolite folinic acid is the most efficient fluoropyrimidine potentiator ${ }^{4}$. As such, the combination of fluoropyrimidines with folinic acid is a standard treatment for colon cancer $^{4}$. A direct intake route has been delineated for several dietary $1 \mathrm{C}$-metabolites including folates, and serine ${ }^{5}$. By contrast, the potential for bacterial uptake routes for $1 \mathrm{C}$ metabolites has not been given much attention despite evidence in its favor ${ }^{8}$. Studies in mammals show bacterially converted dietary para-aminobenzoate-glutamate-one of the two moieties composing THF-in host tissues ${ }^{9,10}$, C. elegans studies demonstrate that $E$. coli mediates the effect of dietary supplementation of folic acid on lifespan ${ }^{11}$, and mouse studies show that bacterially derived serine can affect kidney function ${ }^{12}$. Given that dietary 1C-metabolites, such as folinic acid, are among the most effective potentiators of fluoropyrimidine action, and that the microbiota can alter dietary 1C-metabolites or produce them from dietary precursors, four-way interactions between dietary folates or their precursors, fluoropyrimidines, microbes, and the host, could modulate fluoropyrimidine efficacy and/or toxicity in vivo. In the past several years, C. elegans has been exploited as a model system to study complex drug-microbe-host interactions. Garcia et al. ${ }^{13}$ and Scott et al. ${ }^{14}$ developed a three-way drug-microbe-C. elegans system revealing that microbes mediate chemotherapeutic efficacy in C. elegans. More recently, Pryor et al. ${ }^{15}$ developed a host-microbe-drug-dietary nutrient screen to study the interaction between C. elegans, E. coli, the biguanide metformin, and dietary nutrients. Here we independently developed three and four-way screening strategies to identify and mechanistically dissect the four-way interactions that modulate FUdR toxicity in C. elegans.

First, we investigate the mechanism of toxicity underlying the three-way interaction between FUdR, E. coli, and C. elegans. On the microbe side, we validate that conversion of FUdR into 5fluorouridine monophosphate (FUMP), and not dTMP depletion, contributes to toxicity in C. elegans. On the host side, we define that FUdR toxicity (likely via worm-derivatives of FUMP) targets mitochondrial RNAs and DNA, and that C. elegans die from activation of a lethal mitochondria-to-autophagy axis. Then, we investigate the four-way interaction between dietary metabolites, FUdR, E. coli, and C. elegans. We show that dietary supplementation with thymidine or serine transforms sublethal doses of FUdR (no apparent toxicity) into lethal ones (100\% embryonic lethality) through altering the metabolism of the microbe. However, the mechanisms of action of thymidine and serine are distinct. Thymidine simply enhances the mechanisms driving the three-way interaction, whereas serine acts via enabling dTMP depletion in E. coli and consequently in the host. Most strikingly, dietary serine redefines, or even reverts, the role that host pathways have on executing FUdR toxicity, unveiling sub-phenotypic complexity in four-way diet-drug-microbiota-host interactions.

\section{Results}

FUdR toxicity due to $E$. coli FUMP synthesis, not dTMP depletion. To define whether and how dietary nutrients alter the toxicity of FUdR in C. elegans, it is required first to identify the minimum dose leading to robust toxicity (i.e. $100 \%$ embryonic lethality) for further screens on dietary enhancers and inhibitors of the toxicity. We identified $1 \pm 0.25 \mu \mathrm{g} / \mathrm{mL}$ FUdR as the dose causing $100 \%$ embryonic lethality when worms were cultured on E. coli BW25113 (parental strain of all E. coli mutants used in this study), and $7.5 \pm 2.5 \mu \mathrm{g} / \mathrm{mL}$ FUdR as the dose causing $100 \%$ embryonic lethality when worms were cultured on E. coli HB101 (parental strain of all C. elegans RNAi clones used in this study). We hereinafter refer to these doses as Lth-FUdR (for Lethal FUdR) (Supplementary Fig. 1a).

We then moved on to defining the mechanism of toxicity of Lth-FUdR using a three-way FUdR-E. coli-C. elegans highthroughput screening strategy (summarized in Fig. 1a). We found that $\mathrm{KO}$ of $E$. coli deoA suppresses Lth-FUdR toxicity in $C$. elegans (Fig. 1b, c). DeoA can convert FUdR into Fluorouracil (5FU) (Fig. 1a). Scott et al. ${ }^{14}$ demonstrated that $5-\mathrm{FU}$ is also a prodrug that needs to be converted to be toxic to $C$. elegans. Hence, E. coli DeoA likely carries out one of multiple steps in the conversion of FUdR into the actual toxic derivatives. A reasonable hypothesis would be that the toxic derivative that $E$. coli produces is FUMP, as this would be in line with genetic evidence presented by Garcia et al. ${ }^{13}$. However, single KO of E. coli $u p p$, $u d p$, or $u d k$ was not sufficient to suppress Lth-FUdR toxicity in our screen or follow up retesting (Supplementary Fig. 1b, c). As upp and $u d k$ encode for redundant enzymes capable of converting 5-FU into FUMP, we tested a double KO. Indeed, double KO of E. coli upp and $u d k$ completely suppresses Lth-FUdR toxicity in C. elegans (Fig. 1b, c). These results suggest that E. coli uses the pyrimidine ribonucleotide salvage pathway (i.e. FUdR-to-FUMP conversion pathway) to convert the prodrug FUdR into a derivative toxic to C. elegans. Because nucleotide polyphosphates may not be efficiently taken up by the host, FUMP would be more likely than its downstream derivatives FUDP or FUTP to be the toxic 

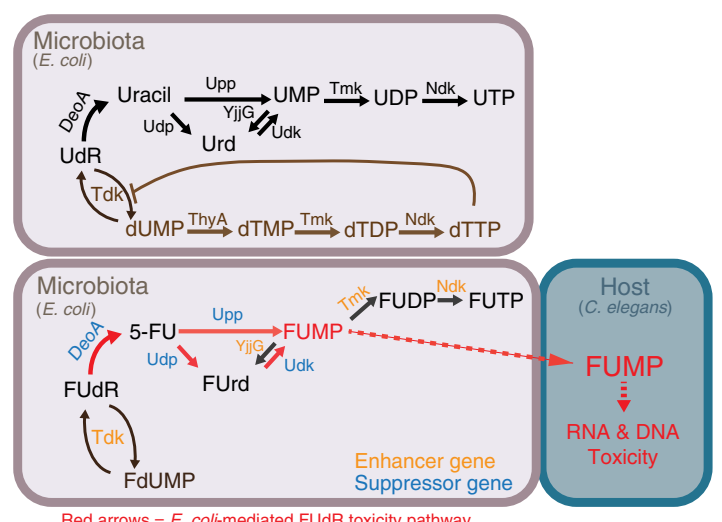

Red arrows $=E$. coli-mediated FUdR toxicity pathway b

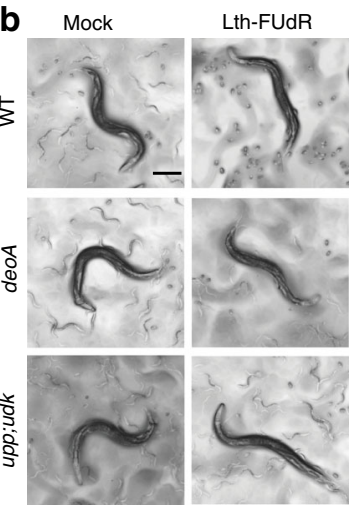

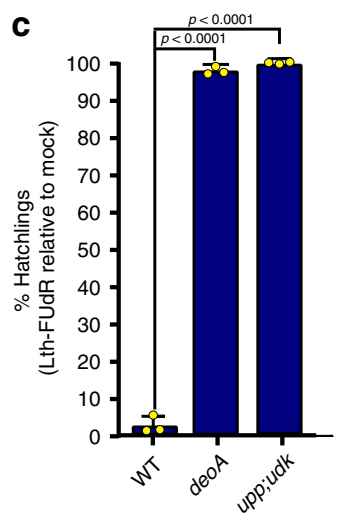

d
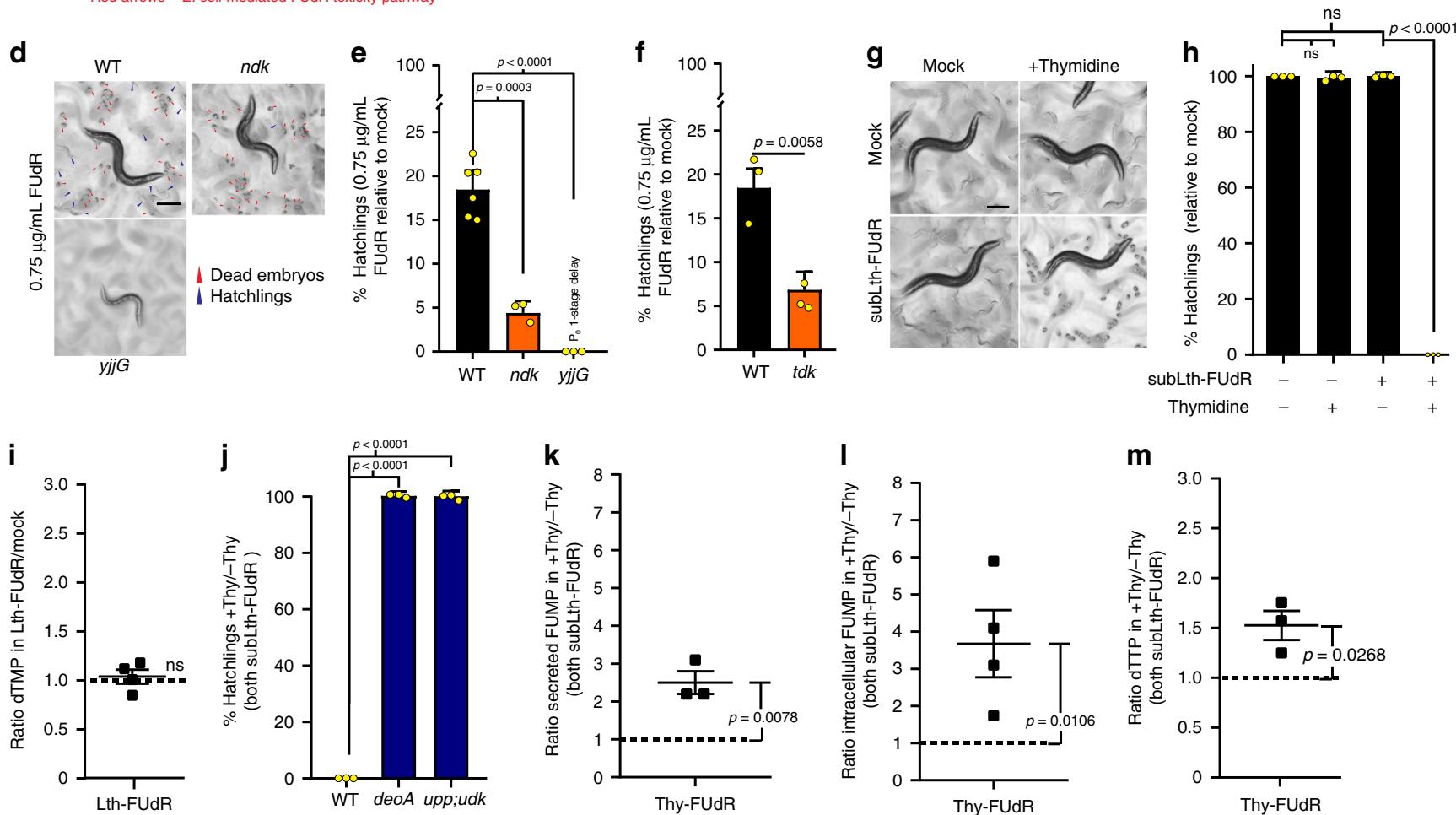

m
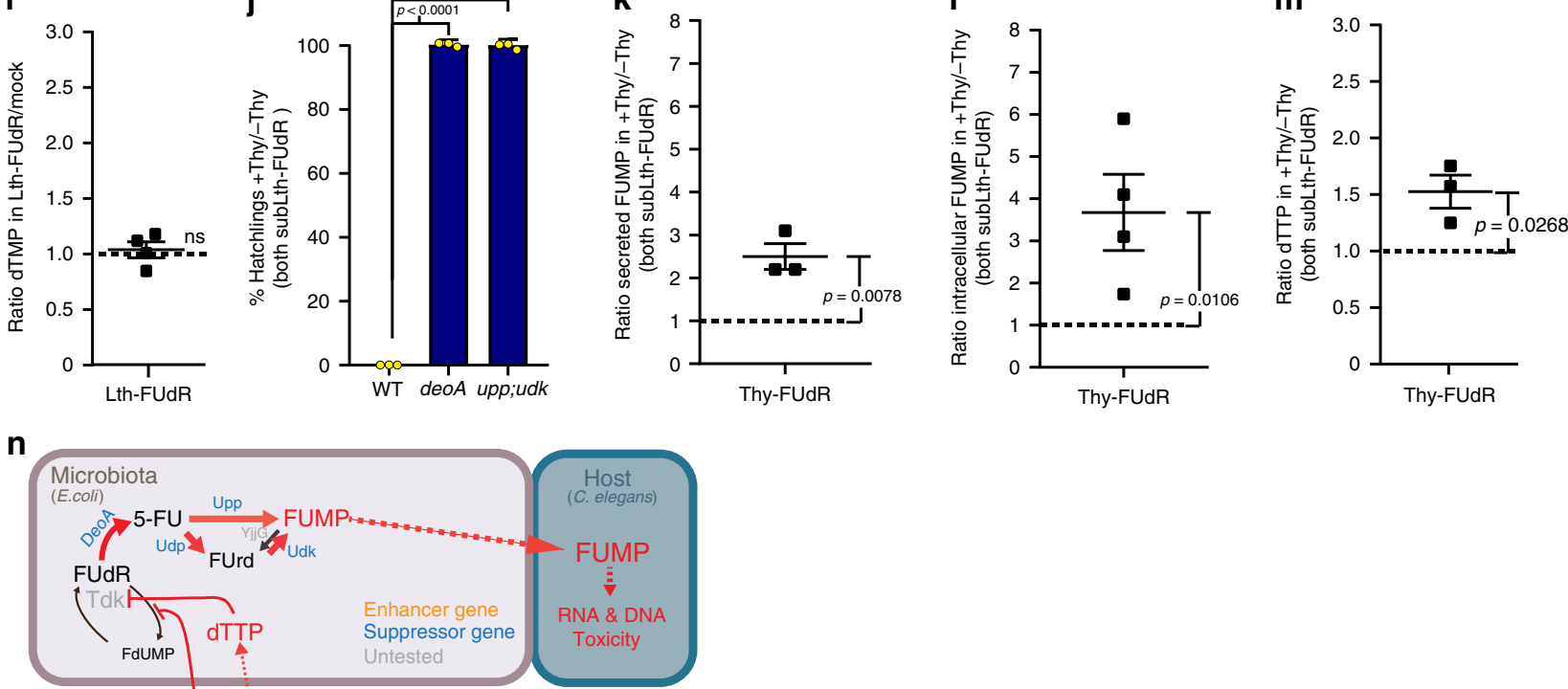

Thymidine

derivative that $E$. coli produces and $C$. elegans takes up. To approximate an answer to this question, we supplemented the plates with UMP (the non-fluorinated analog of FUMP), or the UMP precursors uridine and uracil. We found all three compounds to rescue Lth-FUdR toxicity in C. elegans. By contrast, supplementation with UDP or UTP does not rescue the toxicity (Supplementary Fig. 1d). These results are in line with the notion that nucleotide monophosphates or their unphosphorylated precursors can cross membranes, and hence, could be taken up by the $C$. elegans host while nucleotide polyphosphates would not, and suggest that E. coli-generated nucleotide polyphosphates may not be significant contributors to E. coli-mediated FUdR toxicity in C. elegans. In further support of this notion, chemical inhibition of Tmk (the E. coli enzyme that would produce FUDP) further enhances (instead of suppressing) LthFUdR toxicity (Supplementary Fig. 1e). Similarly, KO of $n d k$ (the E. coli gene encoding the enzyme that would produce FUTP) enhances FUdR toxicity (Fig. 1d, e). Together, the data argue against E. coli-generated FUDP or FUTP being significant contributors to FUdR toxicity in C. elegans. Another E. coligenerated and potentially toxic derivative of FUdR is $5^{\prime}$ fluorouridine (FUrd). However, KO of $y j j G$ (the E. coli gene encoding the enzyme that produces FUrd) enhances FUdR toxicity in C. elegans (Fig. 1d, e). Furthermore, the upp;udk double $\mathrm{KO}(2 \mathrm{KO})$ and the $u p p ; u d k ; u d p$ triple $\mathrm{KO}(3 \mathrm{KO})$ both rescue Lth-FUdR toxicity to the same extent (Supplementary Fig. 1c, f). This result argues against FUrd being a significant contributor to E. coli-mediated FUdR toxicity, because in the 
Fig. 1 Dietary thymidine enhances FUdR-to-FUMP conversion in E. coli. Throughout this figure: \% hatchlings is estimated as [live hatchlings/(live hatchlings + live embryos + dead embryos)] in the condition of interest relative to \% hatchlings in mock of the same $E$. coli or $C$. elegans genotype; $\geq 5$ images per treatment were quantitated; statistical significance was assessed via two-tailed unpaired nonparametric $t$-test. LC-MS data was analyzed using one-tailed ratio $t$-test after ROUT outlier treatment. Data are presented as mean values \pm SEM, scale bars $=200 \mu \mathrm{m}, n=\#$ independent biological replicates. Source data are provided as a Source Data file. a Top: endogenous pyrimidine ribonucleotide salvage pathway (black font) and dTMP de novo synthesis pathway (brown font). Bottom: Model of $E$. coli-mediated FUdR-to-FUMP toxicity pathway. $\mathbf{b}$ Representative images of progeny viability of $C$. elegans treated with mock or Lth-FUdR while cultured on WT (BW25113), deoA, or upp;udk KO E. coli lawns. c Quantification of $\mathbf{b}$ treatments. $n=3$. d Representative images of progeny viability of $C$. elegans treated with mock or $0.75 \mu \mathrm{g} / \mathrm{mL}$ FUdR (lower dose to detect enhancers) while cultured on WT (BW25113), ndk, or yjjG KO E. coli lawns. e Quantification of panel $\mathbf{d}$ treatments. $n=3$. $\mathbf{f}$ Quantification of progeny viability of $C$. elegans treated with mock or $0.75 \mu \mathrm{g} / \mathrm{mL}$ FUdR while cultured on WT (BW25113) or $t d k$ KO E. coli lawns. $n=3$. g Representative images of progeny viability of $C$. elegans treated with FUdR $(0.25 \mu \mathrm{g} / \mathrm{mL}) \pm 5 \mathrm{mg} / \mathrm{mL}$ thymidine. $\mathbf{h}$ Quantification of $\mathbf{g}$ treatments. $n=3$. i LC-MS measurement of dTMP normalized to [ ${ }^{13} \mathrm{C}$, $\left.{ }^{15} \mathrm{~N} 2\right] \mathrm{UMP}$ in $E$. coli treated with Lth-FUdR ( $5 \mu \mathrm{g} / \mathrm{mL}$ ) relative to mock. $n=4$. j Quantification of progeny viability of C. elegans cultured on WT (BW25113), upp; udk, or deoA $\mathrm{KO}$ E. coli lawns treated with subLth-FUdR $(0.25 \mu \mathrm{g} / \mathrm{mL}) \pm 5 \mathrm{mg} / \mathrm{mL}$ thymidine. $n=3$. $\mathbf{k}$ LC-MS measurement of secreted FUMP in $E$. coli supernatants normalized to $\left[{ }^{13} \mathrm{C} 9,{ }^{15} \mathrm{~N} 2\right]$ UMP. $n=3$. I LC-MS measurement of FUMP normalized to $\left[{ }^{13} \mathrm{C} 9,{ }^{15} \mathrm{~N} 2\right] \mathrm{UMP}$ in $E$. coli pellets, $n=4$. $\mathbf{m} \mathrm{LC}-\mathrm{MS}$ measurement of dTTP normalized to $[13 C 9,15 N 2]$ UMP in E. coli pellets, $n=3$. $\mathbf{n}$ Working model of $E$. coli-mediated thymidine-enhanced FUdR toxicity: (1) thymidine-derived dTTP inhibits Tdk, and (2) dietary thymidine competes with FUdR, thereby promoting FUdR-to-FUMP bioconversion.

2KO, FUrd synthesis is favored due to 5 -FU to FUrd conversion, whereas in the $3 \mathrm{KO}$, such conversion is blocked (pathway scheme in Fig. 1a).

Finally, a major candidate to be an E. coli-generated mediator of the toxicity is FdUMP. E. coli-generated FdUMP could act via: (1) inhibiting C. elegans TS post-ingestion; or (2) inhibiting E. coli TS and consequently reducing the availability of thymidine in the $C$. elegans diet because $E$. coli is the main source of nucleotides for C. elegans ${ }^{16}$. An essential step for both mechanisms of action is that E. coli thymidylate kinase (Tdk) converts FUdR into FdUMP. The result of the screen and the follow up retesting showing that $\mathrm{KO}$ of $t d k$ enhances, instead of suppressing, Lth-FUdR toxicity (Fig. 1f) argues against an FdUMP-dependent mechanism of action. In the same line, thymidine supplementation enhances, instead of rescues, Lth-FUdR toxicity (Fig. 1g, h), and LC-MS analyses show that the levels of dTMP did not drop in E. coli treated with a lethal dose of FUdR (Fig. 1i). Thus, the evidence argues against FdUMP directly produced by E. coli, or dTMP depletion in $E$. coli contributing to Lth-FUdR toxicity in $C$. elegans.

In summary, when FUMP synthesis is blocked, we observe abrogation of the toxicity, and when FUMP synthesis or accumulation is promoted, we observe enhanced toxicity. In addition, blocking FdUMP synthesis ( $t d k \mathrm{KO}$ ) enhances the toxicity. Therefore, our data support a model in which FUMP would be the major link between microbe and host fluoropyrimidine metabolism, and host-generated derivatives of FUMP (e.g. FUTP or FdUTP) would promote toxicity in the host.

Thymidine increases $E$. coli-mediated FUdR to FUMP conversion. The observation that in-plate supplementation with thymidine increases FUdR toxicity in C. elegans and that FUdR toxicity in $C$. elegans is mediated by $E$. coli, suggest that dietary thymidine, FUdR, E. coli, and C. elegans may represent an uncharacterized four-way diet-drug-microbe-host interaction. To test the hypothesis that $E$. coli is mediating the potentiating effect of thymidine, we tested whether $E$. coli pretreated with a sublethal dose of FUdR (subLth-FUdR) plus thymidine would be more toxic to C. elegans than E. coli pretreated with subLth-FUdR alone. In this context, worms were not directly exposed to FUdR or thymidine; hence, enhanced toxicity would support the hypothesis that thymidine-enhanced FUdR toxicity is bacterially driven (Experimental setup in Supplementary Fig. 2a). In addition, because the known mechanism of 5 -FU toxicity is production and secretion of FUMP ${ }^{14}$, we separated and independently tested the supernatants and pellets of $E$. coli pretreated with subLth-FUdR plus thymidine. Finally, the filter-sterilized supernatants were seeded on top of triple $u p p, u d p, u d k$ KO lawns to avoid in-plate bacterially driven conversion of the FUdR remaining in the $E$. coli supernatants. We observed that the supernatants and the pellets of $E$. coli pretreated with Lth-FUdR and subLth-FUdR plus thymidine caused embryonic lethality, while the supernatants and pellets pretreated with subLth-FUdR or thymidine alone were not toxic to C. elegans (Supplementary Fig. 2b). Therefore, thymidine-enhanced FUdR toxicity (TEFUdR) is bacterially driven and mediated, at least in part, by a secretable toxic compound. We first tested whether this secretable toxic compound would be the E. coli-generated FUdR-derivative FUMP. In support of this hypothesis, $\mathrm{KO}$ of the gene encoding the E. coli enzymes capable of converting FUdR into FUMP (deoA, or double KO of $u p p$ and $u d k$ ) suppresses TE-FUdR toxicity (Fig. 1j). More directly, we found a $>2$ fold increase in FUMP levels when we compare the supernatants and bacterial pellets of $E$. coli treated with FUdR plus thymidine relative to FUdR alone (Fig. 1k, 1). Therefore, dietary thymidine enhances FUdR toxicity in the C. elegans host through promoting FUdRto-FUMP conversion via the pyrimidine ribonucleotide salvage pathway.

We then asked how thymidine potentiates the toxicity of FUdR. Clues came from the following: (1) KO of the gene encoding Tdk, the enzyme that can convert FUdR into FdUMP, enhances FUdR toxicity (Fig. 1f). This likely occurs because by blocking the conversion of FUdR into FdUMP, we favor the conversion of FUdR into 5-FU and then FUMP (pathway scheme in Fig. 1a); (2) Tdk accepts thymidine as a substrate (ecocyc.org). Hence, thymidine can compete with FUdR and reduce the Tdkmediated conversion of FUdR into FdUMP; and (3) Tdk is subject to end-product inhibition by dTTP (ecocyc.org). As thymidine can serve as a substrate for the synthesis of dTTP, then dietary thymidine could promote end-product inhibition of Tdk. In support of the latter mechanism (but without ruling out the former), we observed increased levels of dTTP in TE-FUdR E. coli (Fig. 1m). Together, the data are consistent with dietary thymidine increasing the toxicity of FUdR via indirectly promoting the conversion of FUdR into FUMP (Working model in Fig. 1n).

Serine increases FUdR toxicity without increasing FUMP levels. After establishing that four-way diet-drug-E. coli-C. elegans interactions such as the thymidine-FUdR-E. coli-C. elegans interaction can be detected and mechanistically dissected in our system, we sought to identify common dietary nutrients that may affect FUdR toxicity. We focused on amino acids (AA) for four reasons: (1) AA derivatives are precursors for the synthesis of 
nucleotides and cofactors needed to synthesize nucleotides ${ }^{17}$; (2) AAs alter chemotherapeutic efficacy in cells in vitro ${ }^{18}$; (3) AAs are among the most highly consumed nutrients by cancer cells ${ }^{19}$; and (4) AA-depleted diets are currently being tested to improve cancer treatment ${ }^{20}$. We tested $19 \mathrm{~L}$-amino acids and glycine for their capacity to promote developmental delay of C. elegans treated with a dose of FUdR that on its own does not affect development. The conditions of the four-way compound screen are depicted in Fig. 2a and are described in the Methods section. Of the $20 \mathrm{AA}$, we found that high doses of tryptophan were toxic on their own whereas glycine and serine increased toxicity in a FUdR-specific manner (Fig. 2b). Serine was a stronger toxicity potentiator than glycine, so we focused on characterizing serineenhanced FUdR toxicity. First, we retested the capacity of serine to potentiate the toxicity of an already lethal dose of FUdR. Hatchlings seeded on E. coli HB101 lawns supplemented with 1.5 $\mathrm{mg} / \mathrm{mL}$ serine alone became fertile adults after $60 \mathrm{~h}$ of incubation at $20^{\circ} \mathrm{C}$, while hatchlings parallelly growing on $12.5 \mu \mathrm{g} / \mathrm{mL} \mathrm{FUdR}$ were sterile adults. However, when we combined serine and FUdR, we observed larval arrest that persisted indefinitely (Fig. 2c), demonstrating that dietary supplementation of serine can potentiate the toxicity of FUdR. Furthermore, hatchlings seeded on E. coli HB101 lawns supplemented with $1.5 \mathrm{mg} / \mathrm{mL}$ serine or a sublethal dose of FUdR $(1 \mu \mathrm{g} / \mathrm{mL}$ FUdR for HB101) yielded $100 \%$ fertile adult C. elegans; however, worms were $100 \%$ sterile when serine and FUdR were combined (Fig. 2d, e). Together, the results show that dietary supplementation of serine can potentiate the toxicity of FUdR across a wide range of doses and toxicity outcomes. Hereafter, we use the term SE-FUdR toxicity to refer to the enhancement of toxicity achieved by combining a sublethal dose of FUdR $(\leq 0.25 \mu \mathrm{g} / \mathrm{mL}$ for BW25113 or $\leq 1 \mu \mathrm{g} / \mathrm{mL}$ for $\mathrm{HB} 101$ ) with dietary serine (Supplementary Fig. 3a).

How does dietary serine enhance FUdR toxicity in C. elegans? A formal possibility is that combining FUdR with serine impairs E. coli growth, thus leading to food scarcity in the worm. However, for all non-screening experiments presented in this study, bacteria were cultured overnight in LB in the absence of additives, and then washed and concentrated in S-buffer to $\mathrm{OD}_{600 \mathrm{~nm}}=20$ before being seeded on nematode growth media (NGM) plates. Furthermore, CFU counting of bacteria harvested from standard NGM or NGM supplemented with serine, subLthFUdR, or subLth-FUdR plus serine shows similar bacterial viability in all conditions (Supplementary Fig. 3b, c). Therefore, E. coli lawn density and growth rates do not appear to explain SEFUdR toxicity in our experimental setup.

We then tested whether SE-FUdR toxicity, like thymidine, was bacterially driven. As with thymidine, we pretreated liquid cultures of E. coli with mock, Lth-FUdR, serine, subLth-FUdR, or subLth-FUdR plus serine, and separated and tested E. coli supernatants and pellets independently (Experimental setup in Supplementary Fig. 4a). We observed toxicity in worms cultured on E. coli pellets pretreated with SE-FUdR (Fig. $2 \mathrm{f}$ and Supplementary Fig. 4b). However, we observed no toxicity in worms exposed to supernatants of E. coli pretreated with SEFUdR (Supplementary Fig. 4b), demonstrating that, unlike thymidine, SE-FUdR toxicity is not driven by secreted E. coli products, and justifying to not further characterize SE-FUdR $E$. coli supernatants in this study. Therefore, SE-FUdR toxicity is bacterially driven, but mainly via an intracellular mechanism. Based on this observation, we can formulate two hypotheses: (1) serine promotes FUdR-to-FUMP conversion but prevents FUMP secretion; or (2) serine promotes a mechanism of toxicity that is distinct from Lth-FUdR and TE-FUdR toxicity. To test the first hypothesis, we measured the levels of 5-FU and FUMP in the E. coli pellets, and found them to be the same in the subLth-FUdR and SE-FUdR conditions (Fig. $2 \mathrm{~g}, \mathrm{~h}$ ), even though aliquots of the bacteria used for metabolite extraction showed the expected 0 and $100 \%$ embryonic lethality, respectively (Supplementary Fig. 5a). This lack of increase of FUMP levels in the SE-FUdR condition is in contrast with the elevated levels of FUMP observed in the TEFUdR condition (Fig. 1k, l), and supports the notion that thymidine and serine potentiate FUdR toxicity through distinct mechanisms.

As the SE-FUdR mechanism of toxicity seems distinct from Lth-FUdR and TE-FUdR, we tested whether fluororibonucleotides other than FUMP, specifically fluorouridine (FUrd), FUDP, or FUTP, were contributing to SE-FUdR. These fluororibonucleotides were below the detection limit of our LC-MS of bacteria or worms treated with subLth-FUdR or SE-FUdR (Supplementary Fig. 5b, and experimental details in Supplementary Note 1). Nevertheless, KO of $y j j G$ or $u d p$, which would reduce FUrd synthesis (pathway scheme in Fig. 1a), does not reduce SE-FUdR toxicity (Supplementary Fig. 5c). Furthermore, blocking the conversion of FUMP-into-FUDP (through chemical treatment with $5^{\prime}$-iodo-UMP) and of FUDP-into-FUTP (through $\mathrm{KO}$ of $n d k)$ further enhances SE-FUdR toxicity, arguing against FUDP or FUTP mediating SE-FUdR toxicity (Supplementary Fig. 5d, e, respectively). Remarkably, these data show that despite the fact that SE-FUdR toxicity is not driven by increased FUMP (Fig. 2h), preventing the conversion of FUMP into FUDP or FUTP further enhances SE-FUdR toxicity. These results prompted us to think about how SE-FUdR toxicity and the FUdR-to-FUMP toxicity pathway interact. We hypothesized that sublethal levels of FUMP toxicity would be necessary to sensitize C. elegans to SE-FUdR toxicity. In support of this hypothesis, we found that the double KO $u p p ; u d k$, and the triple KO upp;udp;udk suppress SE-FUdR toxicity in C. elegans (Fig. $2 \mathrm{i}$, j). Altogether, the results suggest that SE-FUdR is not mediated by increased conversion of FUdR into FUMP (biochemical evidence), or FUDP, FUTP or FUrd (genetic evidence) in E. coli. However, a sublethal level of FUMP toxicity appears to be required to sensitize C. elegans to SE-FUdR toxicity.

E. coli's folate metabolism is required for SE-FUdR toxicity. Having ruled out enhanced FUdR-to-FUMP conversion, we moved to uncover the main bacterially driven mechanism of SEFUdR toxicity. First, we tested whether in-plate supplementation with serine would simply increase the levels of serine in E. coli, and hence, although bacterially driven, SE-FUdR would not require E. coli-mediated conversion of serine. Arguing against this notion, direct measurement of serine and glycine levels in E. coli and C. elegans shows no increase in the levels of these amino acids (Supplementary Fig. 5f, g), even when aliquots of the analyzed bacteria promote enhanced toxicity in the worm (Supplementary Fig. 5a). Hence, we decided to use a four-way E. coli KO suppressor/enhancer screen to molecularly dissect how serine is metabolized in E. coli to enhance FUdR toxicity in C. elegans. To create our E. coli-KO screening library, we used in silico modeling based on the iJO1366 E. coli metabolic model to search for all $E$. coli genes within two-metabolic steps from the homologs of the mammalian fluoropyrimidine metabolic pathways (gene list in Supplementary Table 1, and 96-well screen setup in Fig. 3a). The four-way high-throughput screen identified $29 \mathrm{E}$. coli genes altering SE-FUdR toxicity in C. elegans (Supplementary Table 2). Genes belonging to metabolic pathways enriched among the hits were retested in $6 \mathrm{~cm}$ NGM plates. Twelve primary hits were validated using the following criteria: $(1)>$ or $<10 \%$ hatchlings than worms seeded on WT E. coli; (2) p-value $<0.05$; and (3) growth of the E. coli $\mathrm{KO}$ clone in \pm serine \pm FUdR did not correlate with its enhancer or suppressor phenotype (Supplementary 
a

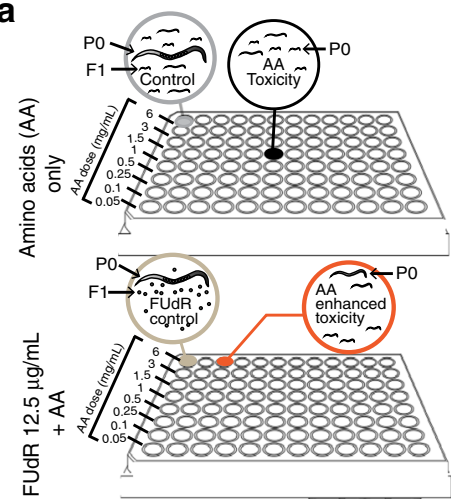

d

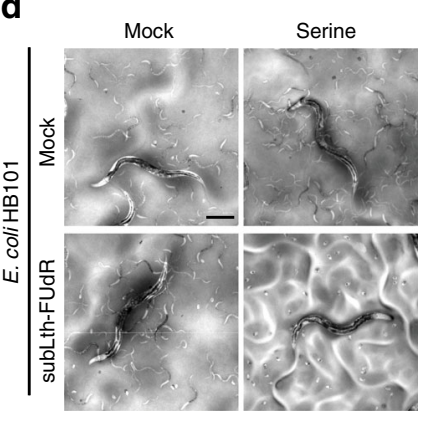

b

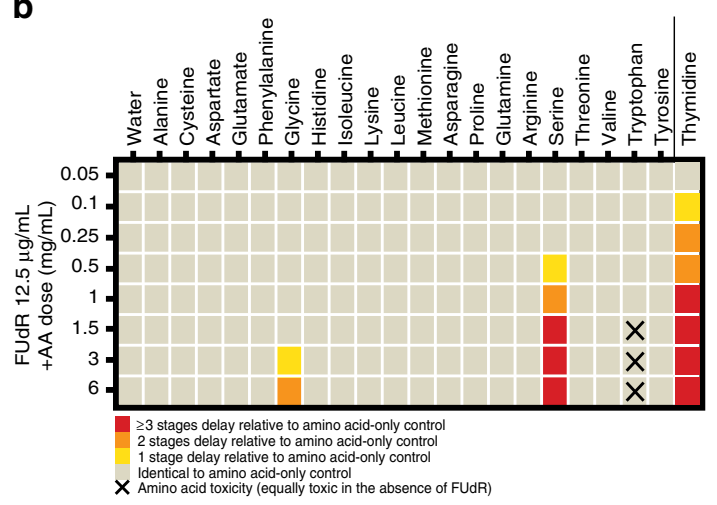

e

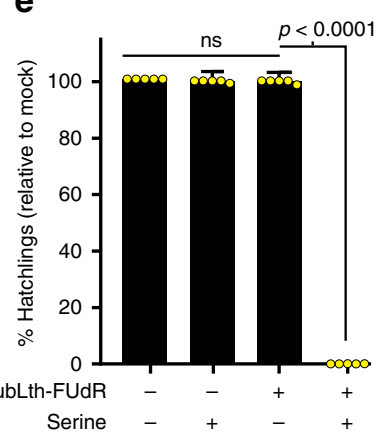

C
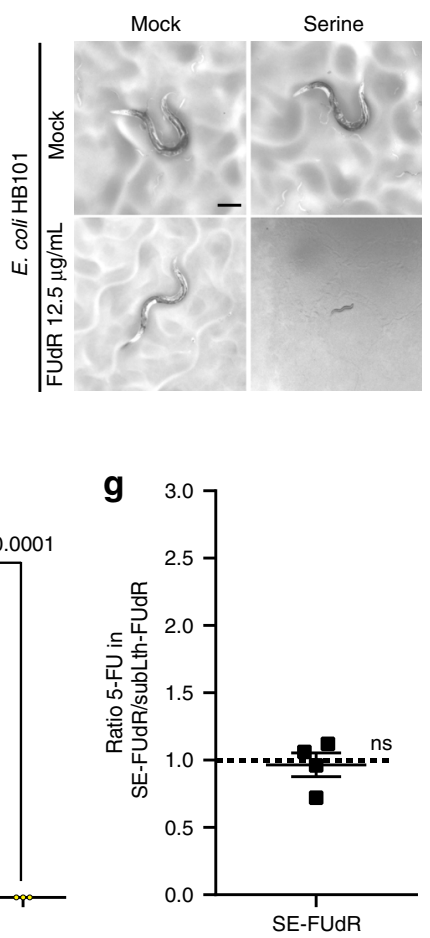
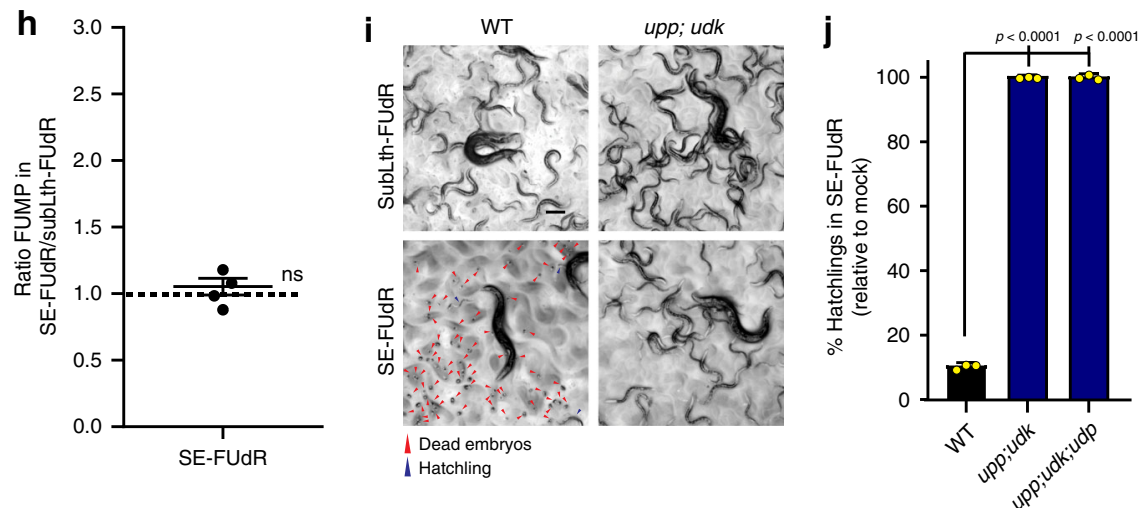

Fig. 2 Dietary serine enhances FUdR toxicity but not FUdR-to-FUMP conversion. Throughout this figure: \% hatchlings and LC-MS data were analyzed as described in Fig. 1. Statistical significance was assessed via two-tailed unpaired nonparametric $t$-test for \% hatchlings quantification. LC-MS data were analyzed using one-tailed ratio $t$-test after ROUT outlier treatment. Data are presented as mean values \pm SEM, scale bars $=200 \mu m, n=\#$ independent biological replicates. Source data are provided as a Source Data file. a Screen setup to search for dietary amino acids (AAs) that enhance FUdR toxicity (cause developmental delay). Worms cultured on E. coli HB101 were treated with $12.5 \mu \mathrm{g} / \mathrm{mL}$ FUdR from the L1 stage \pm serial dilutions of AAs $(0.05-6 \mathrm{mg} / \mathrm{mL})$. AA-only wells were included to detect AA toxicity. Wells were scored after $60 \mathrm{~h}$ at $20^{\circ} \mathrm{C}$, when FUdR-only wells show $100 \%$ embryonic lethality but no developmental delay. b Heat map representing degree of developmental delay caused by supplemented AAs. Color and symbol key depicted below. Thymidine is a positive control. c Representative images of targeted validation of developmental delay induced by co-administration of $12.5 \mu \mathrm{g} / \mathrm{mL}$ FUdR with $1.5 \mathrm{mg} / \mathrm{mL}$ of serine. Images taken after $60 \mathrm{~h}$ of incubation at $20^{\circ} \mathrm{C} . n>3$. d Representative images of progeny viability of $C$. elegans cultured on HB101 and treated from L1 with mock, subLth-FUdR $(1 \mu \mathrm{g} / \mathrm{mL}$ FUdR, which is sublethal because using HB101), $1.5 \mathrm{mg} / \mathrm{mL}$ serine, or subLthFUdR plus serine. $n>10$. e Quantification of \% hatchlings relative to mock of treatments represented in $\mathbf{d} . n=5$. $\mathbf{f}$ Quantification of \% hatchlings in worms exposed to lawns of $E$. coli pretreated "in tube" with \pm subLth-FUdR \pm serine. In this setup, worms are not directly exposed to FUdR or serine (Supplementary Fig. 4). $n=3 . \mathbf{g}$ LC-MS measurement of intracellular 5-FU relative to internal standard (IS) $[1,3-15 \mathrm{~N} 2]$ Uracil in $E$. coli treated with subLth-FUdR plus serine compared to subLth-FUdR. $n=4$. $\mathbf{h}$ LC-MS measurement of intracellular FUMP relative to internal standard (IS) $\left[{ }^{13} \mathrm{C} 9,{ }^{15} \mathrm{~N} 2\right] \mathrm{UMP}$ in $E$. coli treated with subLth-FUdR plus serine compared to subLth-FUdR. $n=4$. i Representative images of progeny viability of C. elegans cultured on WT (BW25113) or upp; udk $\mathrm{KO}$ E. coli lawns treated from $\mathrm{L} 1$ with subLth-FUdR $(0.25 \mu \mathrm{g} / \mathrm{mL}) \pm$ serine. $\mathbf{j}$ Quantification of $\%$ hatchlings relative to mock of treatments represented in i and the triple E. coli KO upp;udp;udk. $n=3$. 
a

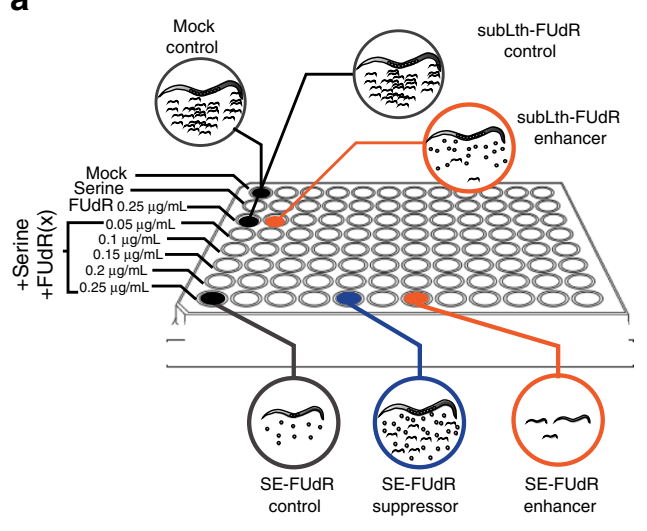

C

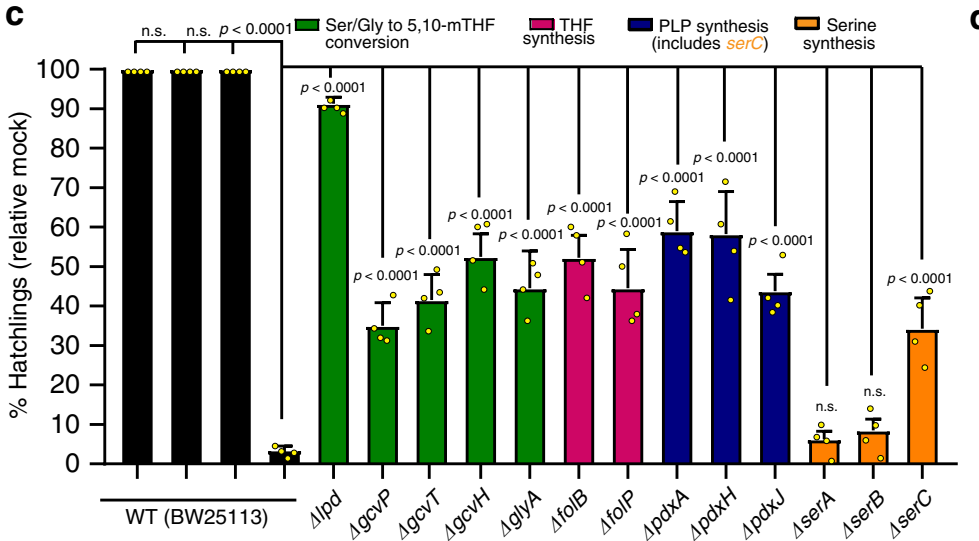

b $\quad B W \quad 10 d$
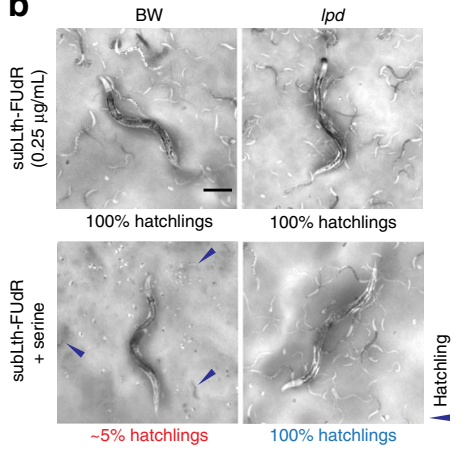

subLth-FUdR

Serine -

Fig. 3 SE-FUdR toxicity requires $\boldsymbol{E}$. coli's folate and pyridoxal phosphate synthesis pathways. Throughout this figure: statistical significance was assessed via two-tailed unpaired nonparametric $t$-test for $\%$ hatchlings quantification. Data are presented as mean values \pm SEM, scale bars $=200 \mu m$, $n=$ \# independent biological replicates. Source data are provided as a Source Data file. a Setup of four-way E. coli KO screen for mediators of serineenhanced FUdR toxicity. Screening was carried out in the BW25113 background in triplicate at $25^{\circ} \mathrm{C}$ in 8 conditions: (1) mock, (2) serine $1.5 \mathrm{mg} / \mathrm{mL}$, (3) subLth-FUdR $0.25 \mu \mathrm{g} / \mathrm{mL}$, and (4-8) serine $1.5 \mathrm{mg} / \mathrm{mL}$ plus subLth-FUdR from 0.05 to $0.25 \mu \mathrm{g} / \mathrm{mL}$ (lower doses included to detect toxicity enhancers). Each column of the 96 -well plate corresponds to a different $E$. coli KO. Developmental stage and progeny viability were scored. $\mathbf{b}$ Representative images of validation of the toxicity-suppressor effect of knocking down $E$. coli lpd, making evident that SE-FUdR toxicity is bacterially driven. $n=4$. $\mathbf{c}$ Effect of the $E$. coli suppressors of SE-FUdR toxicity on progeny viability (\% hatchlings). Images and data were analyzed as described in Fig. 1. $n=4$. $\mathbf{d}$ Working model of how dietary serine promotes synthesis of 5,10-mTHF in E. coli. Color codes of suppressor gene names are consistent with c. Serine relevant actions (depicted in

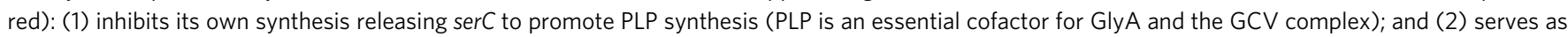
a substrate for the synthesis of 5,10-mTHF directly via GlyA and indirectly via the GCV complex.

Fig. 6). Among the SE-FUdR toxicity suppressors, $l p d$ emerged as the strongest hit (Fig. 3b, c). Lpd encodes E. coli lipoamide dehydrogenase, which is part of three multicomponent enzymatic complexes: pyruvate dehydrogenase, 2-oxoglutarate dehydrogenase, and the glycine cleavage complex (GCV). The GCV complex is composed of Lpd, GcvP, GcvT, and GcvH, and catalyzes the conversion of glycine into 5,10-methylene-tetrahydrofolate $(5,10-\mathrm{mTHF})$. KO of $g c v P, g c v T$, and $g c v H$, also suppresses SE-FUdR toxicity (Fig. 3c), favoring the notion that Lpd would contribute to SE-FUdR toxicity as a component of the GCV complex. However, $l p d$ is a stronger suppressor than the $g c v$ genes. Several distinctions exist between Lpd and the Gcv proteins. For instance, the $g c v$ genes are part of a single operon transcribed by Fnr, whereas lpd is encoded as a single gene and is transcribed by Crp (ecocyc.org). In addition, Lpd is necessary for the activation of the Gcv proteins ${ }^{21}$. However, given the stronger suppressor phenotype of $l p d$ relative to the $g c v$ genes, roles for Lpd beyond the GCV complex cannot be ruled out. Nevertheless, KO of glyA, which encodes the enzyme that converts serine into glycine and 5,10-mTHF, also suppresses SEFUdR toxicity (Fig. 3c, and pathway scheme in Fig. 3d). Together,
GlyA and the GCV complex can convert serine into 5,10-mTHF (pathway scheme in Fig. 3d); and therefore, these suppressors suggest that 5,10-mTHF may have an important role in mediating SE-FUdR toxicity. Pointing to the same direction, KO of folP and folB, suppresses SE-FUdR toxicity (Fig. 3c). FolP and FolB synthesize tetrahydrofolate, which is the precursor of 5,10-mTHF (pathway scheme in Fig. 3d). Further, both the GCV complex and GlyA require the cofactor vitamin $\mathrm{B} 6$ (pyridoxal-5'-phosphate or PLP) to synthesize 5,10-mTHF (ecocyc.org). Hence, it is relevant that $\mathrm{KO}$ of the PLP-synthesis genes $p d x A, p d x J, p d x H$, and $\operatorname{ser} C$ suppress SE-FUdR toxicity (Fig. 3c). Of note, the other enzymatic complexes containing Lpd, namely pyruvate dehydrogenase and 2-oxoglutarate dehydrogenase, do not use PLP as a cofactor, further favoring the notion that the main contribution of Lpd to SE-FUdR toxicity would be through its role as a component of the GCV complex.

Additional insight into how serine potentiates the toxicity of FUdR is garnered from the role that the serine-synthesis pathway has in SE-FUdR toxicity. SerA, SerB, and SerC are essential for de novo synthesis of serine in E. coli (ecocyc.org). However, only $\mathrm{KO}$ of $\operatorname{serC}$ suppresses SE-FUdR toxicity in C. elegans (Fig. 3c). 
Distinctively, SerC is involved in PLP synthesis, whereas SerA and SerB only contribute to serine synthesis (pathway scheme in Fig. 3d). Also importantly, SerA is subject to end-product inhibition by serine. Therefore, the data suggest that serine promotes SE-FUdR toxicity via promoting the synthesis of 5,10mTHF. Serine would promote 5,10-mTHF synthesis through at least two mechanisms (Fig. 3d): (1) inhibiting its own synthesis (via SerA inhibition), thereby freeing SerC to synthesize PLP; and (2) serving as a substrate for the synthesis of 5,10-mTHF via GlyA and the GCV complex. The capacity to free SerC via end-product inhibition distinguishes serine from glycine, and could underlie the observation that dietary glycine is a weaker potentiator of FUdR toxicity than serine (Fig. 2b). Altogether, dietary serine promotes FUdR toxicity in C. elegans through a bacterially driven mechanism that involves conversion of serine and glycine into 5,10-mTHF, and not increased bacterial conversion of FUdRinto-FUMP or accumulation of serine or glycine.

Dietary serine reduces $E$. coli's and hence $C$. elegans' dTMP pool. The observation that $5,10-\mathrm{mTHF}$ synthesis in $E$. coli is essential to SE-FUdR toxicity points toward the best characterized mechanism of fluoropyrimidine toxicity: the formation of a ternary complex composed of FdUMP, 5,10-mTHF, and thymidylate synthase (TS) that inhibits TS function ${ }^{4}$. Importantly, mammalian evidence suggests that 5,10-mTHF is the main limiting factor in the formation of this inhibitory complex ${ }^{4}$. Hence, the next step was to define whether E. coli-generated 5,10-mTHF might act through inhibition of the worm TS or E. coli TS, or both. If worm TS is inhibited, serine would promote 5,10-mTHF synthesis in E. coli, elevating the levels of 5,10-mTHF in the $C$. elegans diet, and enabling the inhibition of C. elegans' TS (TYMS1). Arguing against this scenario, 5,10-mTHF is known to poorly cross membranes, 5 , and strong reduction of C. elegans TS expression through RNAi against C. elegans tyms-1 (Supplementary Fig. 7a) does not enhance FUdR toxicity (Fig. 4a). Using a similar rationale, if dietary serine enhances FUdR toxicity mainly by enabling the inhibition of $E$. coli's TS, culturing worms on TS-deficient E. coli (thyA KO) should enhance their sensitivity to sublethal doses of FUdR. Indeed, although feeding thy A KO bacteria or treating with subLth-FUdR alone is not toxic to $C$. elegans, feeding thy $\mathrm{KO}$ bacteria in the presence of sublethal levels of FUdR leads to $>90 \%$ embryonic lethality in C. elegans (Fig. 4b, c), phenocopying SE-FUdR. Furthermore, the enhanced FUdR toxicity observed in worms fed the thy A KO cannot be further enhanced by dietary serine (Fig. $4 \mathrm{~d}$, e), suggesting that thy $\mathrm{A} \mathrm{KO}$ and dietary serine enhance FUdR toxicity through the same mechanism (see rationale of conditions for this experiment in Supplementary Note 2). Another prediction of the scenario in which dietary serine enables the inhibition of $E$. coli's TS is that SE-FUdR toxicity would depend upon E. coli capacity to convert FUdR into FdUMP, a reaction carried out by Tdk. In line with this prediction, we found that $\mathrm{KO}$ of E. coli tdk partially suppresses SE-FUdR toxicity (Fig. 4f). The observed modest suppression is expected because $\mathrm{KO}$ of $t d k$ would simultaneously enhance FUdR-to-FUMP bioconversion (Fig. If and pathway scheme in Fig. 1n).

The above observations are in line with a model in which dietary serine, via promoting the synthesis of 5,10-mTHF, enables the inhibition of E. coli ThyA, thereby reducing the levels of dTMP in the C. elegans diet. To test this model directly, we measured dTMP levels in E. coli. We found that E. coli treated with subLth-FUdR plus serine show reduced dTMP levels (Fig. $4 \mathrm{~g}$ ). Based on the tyms-1 versus thyA experiments described above (Fig. 4a-e), we proposed that C. elegans's dTMP pool would be limited by E. coli's ability to provide dTMP. Supporting this, we found that $C$. elegans's dTMP levels are reduced in the SE-FUdR condition, and that single KO of E. coli's lpd suppresses this reduction (Fig. 4g). Altogether, the data demonstrate that $E$. coli-mediated conversion of serine/glycine into 5,10-mTHF promotes a reduction of the dTMP pool in E. coli, and consequently in C. elegans.

We next reasoned that if reduced dTMP availability in the $C$. elegans diet is the main SE-FUdR toxicity mechanism, and not a mere correlation, dietary supplementation with dTMP should suppress SE-FUdR toxicity. To test this prediction, we used the complex experimental setup depicted in Fig. 4h, and described in detail in Supplementary Note 3. A key aspect of this experimental setup is that $5^{\prime}$-fluoroorotic acid (5-FO), a source of FUMP that does not need Upp/Udk-mediated conversion, is used to sensitize C. elegans to SE-FUdR toxicity. The first important observation we made is that serine enhances fluoropyrimidine toxicity in a $u p p ; u d k$ double KO background (Fig. $4 \mathrm{i}, \mathrm{j}$ ). This observation is consistent with the notion that FUMP is important to sensitize to SE-FUdR toxicity but that increased flux through the pyrimidine salvage pathway is not how serine enhances toxicity (Fig. 2h). Most significantly, dTMP supplementation suppresses SE-FUdR toxicity (Fig. 4i, j, additional control images in Supplementary Fig. 7b). Therefore, the LC-MS data demonstrate that dietary serine inhibits the production of dTMP in bacteria and that in turn reduces the dTMP pool in C. elegans, and the dTMP-rescue data demonstrate that scarce dietary thymidine is a major contributor to death in C. elegans.

To go one step further and test whether precursors for the synthesis of 5,10-mTHF may limit thymidine-depletion in our experimental setup, we exposed worms to a combination of: (1) 5-FO as the source of sublethal levels of FUMP, (2) $2.5 \mu \mathrm{g} / \mathrm{mL}$ FdUMP, and (3) deoA E. coli mutant as the microbe. In this condition, high levels of FdUMP can accumulate because we provide $\sim 10$ fold more FdUMP $(2.5 \mu \mathrm{g} / \mathrm{mL})$ than the amount of FUdR we normally use to characterize SE-FUdR $(0.25 \mu \mathrm{g} / \mathrm{mL}$ FUdR), and because the $\mathrm{KO}$ of $d e o A$ prevents the conversion of FUdR into 5-FU or FUMP. Nevertheless, despite the expected increase in FdUMP levels, we see no toxicity in C. elegans (Supplementary Fig. 7c). However, supplementing these plates with as little as $150 \mu \mathrm{g} / \mathrm{mL}$ of serine leads to $>70 \%$ lethality, and from there the severity of the toxicity correlates with the amount of serine added to the system (Supplementary Fig. 7c).

Altogether we propose a model in which dietary serine enhances FUdR toxicity through promoting the synthesis of 5,10-mTHF, and with that the formation of the TS inhibitory complex, which results in reduced dTMP production in E. coli and thymidine-less death in worms (Fig. 4k). Microbe-mediated thymidine starvation in C. elegans can be triggered genetically via $\mathrm{KO}$ of $E$. coli thyA or dietarily via supplementation of serine, and likely glycine, in combination with FUdR or FdUMP. Although SE-FUdR toxicity does not act through enhancing the known FUdR-to-FUMP toxicity pathway, it does require FUMP to sensitize the worm to thymidine-less death. This is in line with a previous study demonstrating that nucleotide imbalance in the microbe alone is insufficient to promote toxicity in C. elegans ${ }^{16}$. Together, the data show the critical role that four-way interactions can have in fluoropyrimidine toxicity in the host. The results also highlight the need to control animal husbandry conditions to make generalized conclusions about the role microbe and host pathways have in the response to drugs.

The host distinctively responds to Lth-FUdR and SE-FUdR toxicity. Although the phenotypic outcomes of treatment with Lth-FUdR and SE-FUdR are similar, namely embryonic lethality at low doses and developmental delay at higher doses, the 
a

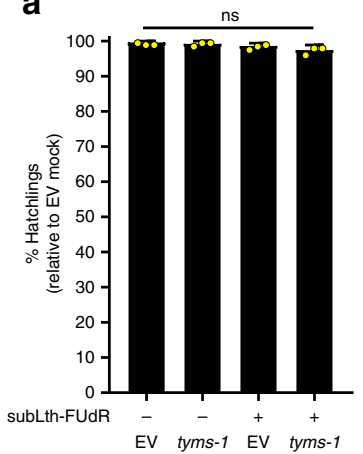

b

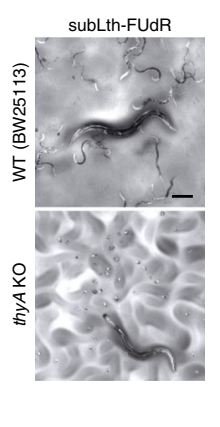

C

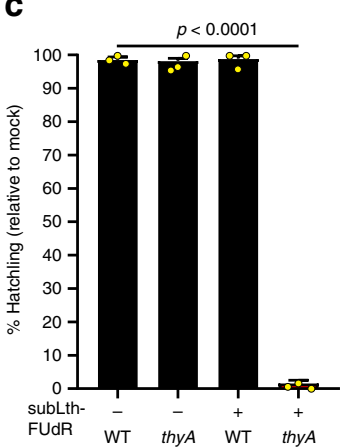

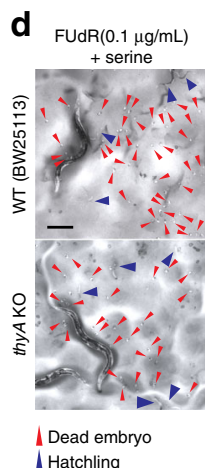

e

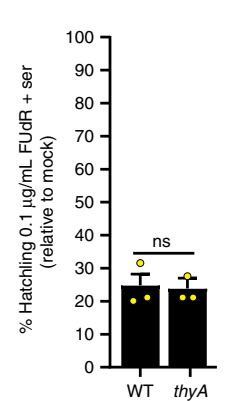

f

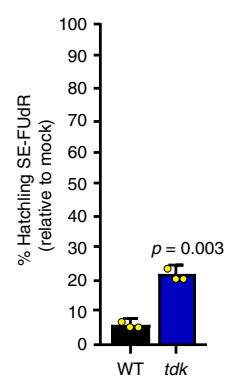

g

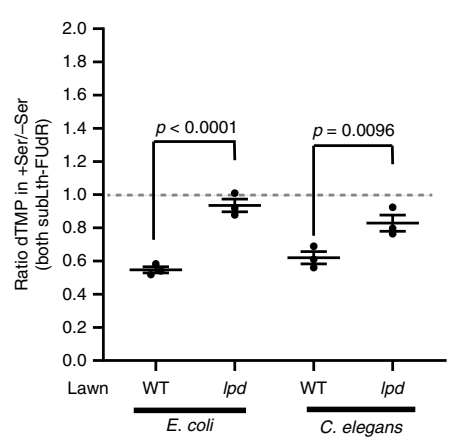

h

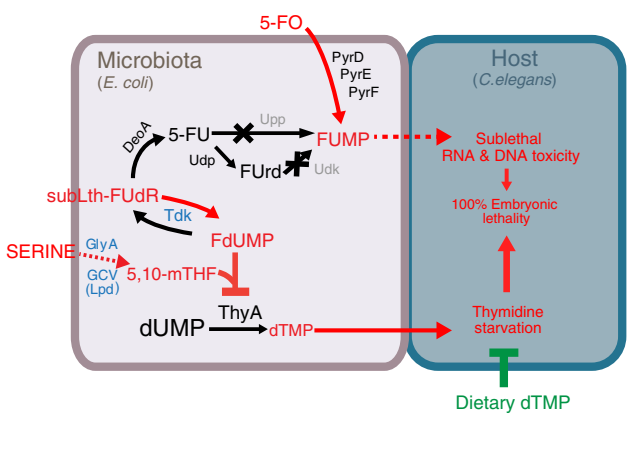

i lawn: upp;udk(2KO)

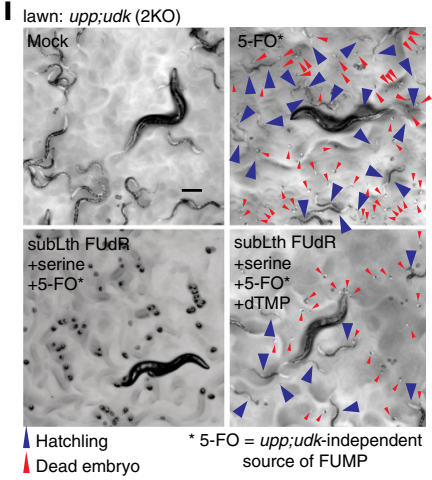

j

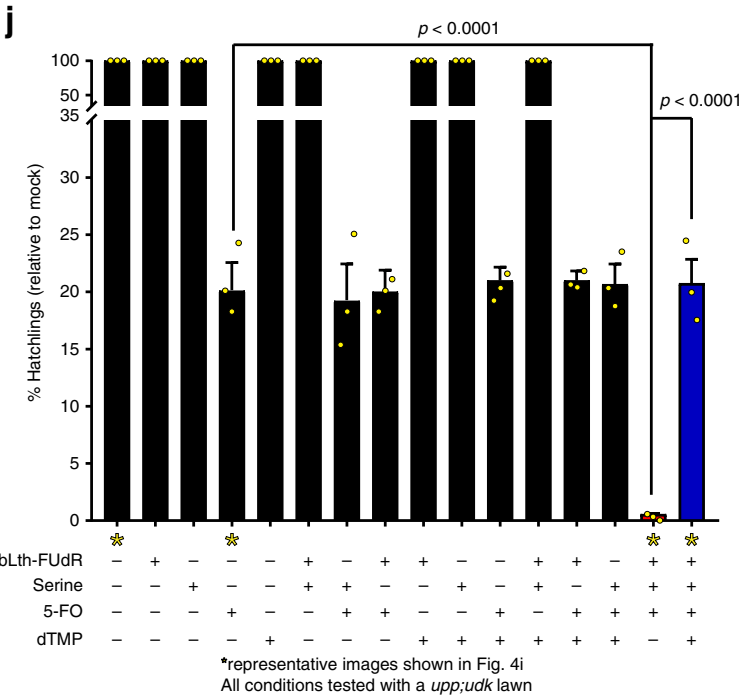

All conditions tested with a upp;udk lawn

k

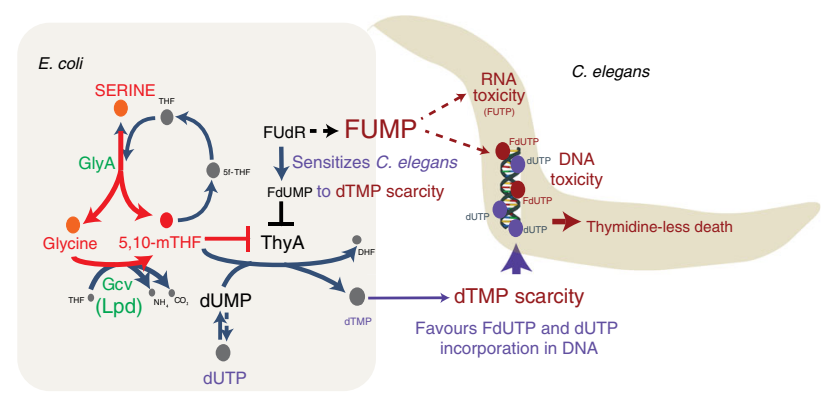

Fig. 4 SE-FUdR promotes dTMP depletion in E. coli and C. elegans. Throughout this figure: \% hatchlings and LC-MS data were analyzed as described in Fig. 1. Statistical significance was assessed via two-tailed unpaired nonparametric $t$-test for $\%$ hatchlings quantification. LC-MS data were analyzed using one-tailed ratio $t$-test after ROUT outlier treatment. Data are presented as mean values \pm SEM, scale bars $=200 \mu \mathrm{m}$. $n=\#$ independent biological replicates. Source data are provided as a Source Data file. a Quantification of progeny viability of $C$. elegans exposed to subLth-FUdR $(1 \mu \mathrm{g} / \mathrm{mL})$ while cultured on EORB1 (RNAi-competent derivative of HB101) carrying empty RNAi empty vector (EV) or RNAi against tyms-1. $n=3$. See EORB1 strain development in Methods and Supplementary Fig. 8. b Representative images of progeny viability of C. elegans exposed to subLth-FUdR (0.25 $\mu \mathrm{g} / \mathrm{mL})$ while cultured on WT (BW25113) or thyA KO E. coli lawns. c Quantification of $\mathbf{b}$ treatments. $n=3$. $\mathbf{d}$ Representative images of progeny viability of $C$. elegans exposed to subLth-FUdR $(0.1 \mu \mathrm{g} / \mathrm{mL}$ ) + serine $1.5 \mathrm{mg} / \mathrm{mL}$ while cultured on WT (BW25113) or thyA KO E. coli lawns. e Quantification of $\mathbf{d}$ treatments. $n=$ 3. $\mathbf{f}$ Quantification of progeny viability of $C$. elegans exposed to subLth-FUdR \pm serine while cultured on WT (BW25113) or $t d k$ KO E. coli lawns. $n=3$. g LC-MS measurement of dTMP normalized to $\left[{ }^{13} \mathrm{C} 9,{ }^{15} \mathrm{~N} 2\right]$ UMP (Norm dTMP) in E. coli WT (BW25113) and Ipd KO, and C. elegans cultured in these two E. coli strains. The ratio Norm dTMP in SE-FUdR / Norm dTMP in subLth-FUdR is depicted for each treatment. $n=3$. $\mathbf{h}$ Setup of dTMP-rescue experiment: (1) upp; udk double KO lawn avoids enhanced-FUMP toxicity otherwise driven by thymidine; (2) 5'-fluoroorotic acid (5-FO) as a source of FUMP; (3) subLthFUdR + serine to promote SE-FUdR; (4) $\pm d T M P$ to test rescue of SE-FUdR toxicity. i Representative images of progeny viability of $C$. elegans cultured on upp; udk double KO E. coli lawns $\pm 5-F O, \pm S E-F U d R(0.25 \mu \mathrm{g} / \mathrm{mL}$ FUdR and $1.5 \mathrm{mg} / \mathrm{mL}$ serine $), \pm d T M P(1.5 \mu \mathrm{g} / \mathrm{mL})$ showing dTMP rescues SE-FUdR toxicity. j Quantification of treatments in $\mathbf{i}$ (denoted with asterisks) and other controls. $n=3$. $\mathbf{k}$ Working model of SE-FUdR toxicity. Through promoting $5,10-\mathrm{mTHF}$ synthesis, dietary serine enables FdUMP-mediated inhibition of $E$. coli TS (ThyA). The consequent scarcity of dietary dTMP then exacerbates the toxic effect of sublethal FUdR, leading to DNA toxicity, and death of the worm. 
a
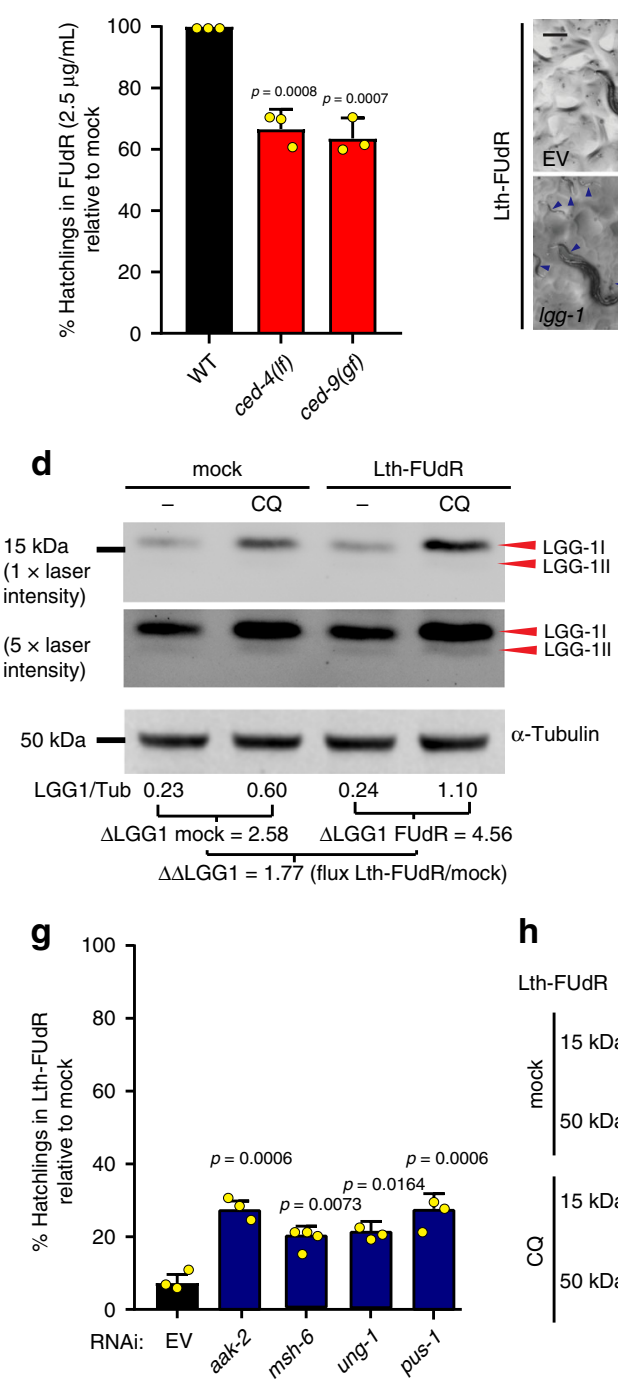

b

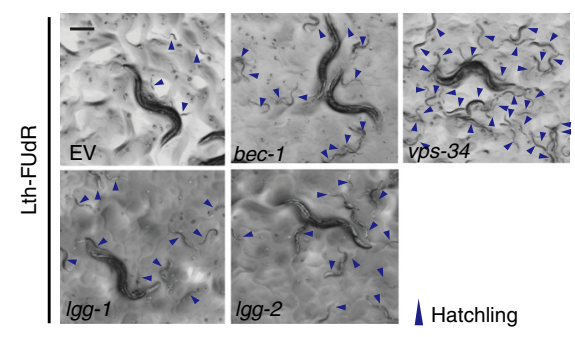

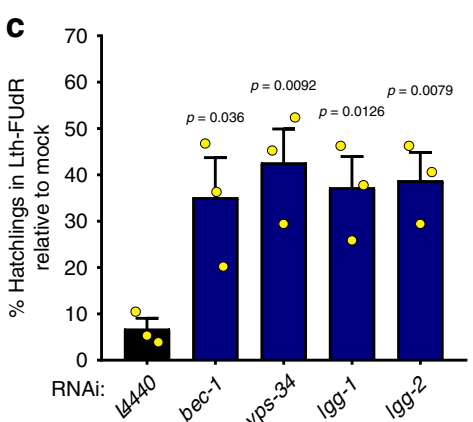

e

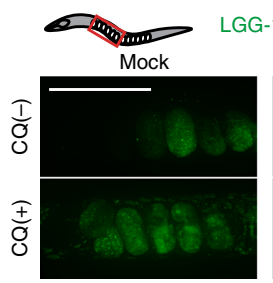

LGG-1::pH-sensitive GFP Lth-FUdR
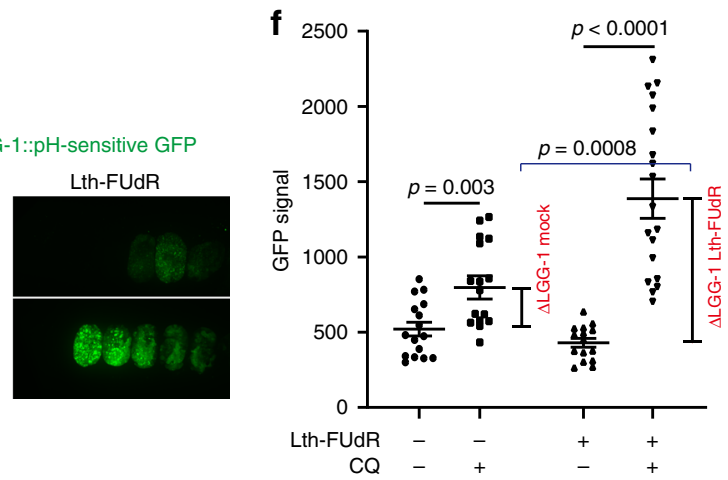

i h Lth-FUdR 을

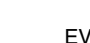

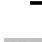
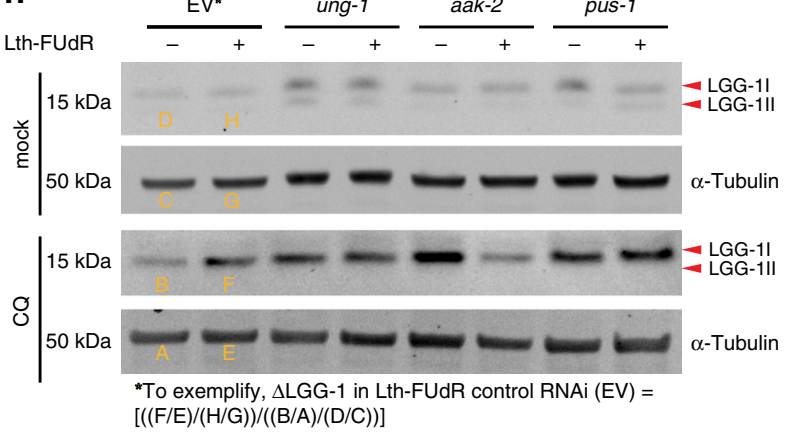

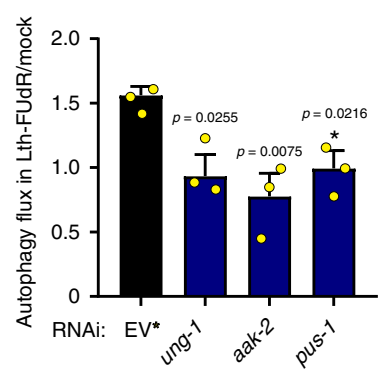

Fig. 5 Lth-FUdR activates autophagic cell death in C. elegans. Throughout this figure: \% hatchlings was analyzed as described in Fig. 1. Statistical significance was assessed via two-tailed unpaired nonparametric $t$-test for $\%$ hatchlings quantification. Statistical significance for western blotting ratio was assessed via one-tailed ratio $t$-test. Data are presented as mean values \pm SEM, $n=\#$ independent biological replicates. Source data are provided as a Source Data file. a Quantification of progeny viability of WT, ced-4(n1162), and ced-9(n1950) mutant C. elegans exposed to $2.5 \mu \mathrm{g} / \mathrm{mL}$ FUdR (lower dose of FUdR used to enable detection of enhancers). $n=3$. $\mathbf{b}$ Representative images of progeny viability of $C$. elegans exposed to Lth-FUdR while cultured on EORB1 EV or autophagy RNAi clones. Scale bar $=200 \mu \mathrm{m}$. c $\%$ hatchling quantification of treatments represented in $\mathbf{b}$. $n=3$. d Representative $\alpha \mathrm{LGG}-1$ western blotting of worms cultured on EORB1 lawn \pm Lth-FUdR $(7.5 \mu \mathrm{g} / \mathrm{mL}) \pm 8 \mathrm{~h}$ exposure to $20 \mathrm{mM}$ chloroquine (lysosomal inhibitor). Two different exposures of $\alpha \mathrm{LGG}-1$ blot are depicted. Autophagy flux estimation and data interpretation described in main text and Methods. $n=10$. e In vivo imaging of embryos expressing LGG-1::GFP(pH-sensitive) treated with \pm Lth-FUdR $(7.5 \mu \mathrm{g} / \mathrm{mL})$ and $\pm 8 \mathrm{~h}$ of $20 \mathrm{mM}$ chloroquine. Scale bar $=100 \mu \mathrm{m}$. $\mathbf{f}$ Quantification of GFP signal of treatments represented in e, two-tailed, unpaired, nonparametric $t$-test. LGG-1::GFP data acquisition, analyses, and interpretation described in main text and Methods. Unpaired nonparametric one-tailed $t$-test was used to singly compare average GFP signal in $(+)$ CQ to $(-)$ CQ (denoted with black brackets and asterisks), and one-tailed ratio $t$-test was used to compare $\Delta$ LGG-1 ratios (denoted with blue brackets and asterisks). $n=3$. g Quantification of progeny viability of $C$. elegans exposed to Lth-FUdR $(7.5 \mu \mathrm{g} / \mathrm{mL})$ while cultured on EORB1 EV or RNAi against aak-2, msh-6, ung-1, or pus1. $n=3$. $\mathbf{h}$ Representative $\alpha \mathrm{LGG}-1$ western blotting of worms cultured on EORB1 EV or RNAi against ung- 1 , aak- 2 or pus $-1 \pm \mathrm{Lth}-\mathrm{FUdR}(7.5 \mu \mathrm{g} / \mathrm{mL}) \pm 8 \mathrm{~h}$ of $20 \mathrm{mM}$ chloroquine. Approach, data analyses, and interpretation described in main text and Methods. $\mathbf{i}$ Autophagy flux quantification as depicted in $\mathbf{h}$, and described in Methods. $n=3$.

microbial mechanisms leading to these outcomes are distinct. Thus, we next sought to investigate whether the host response to Lth-FUdR and SE-FUdR at a sub-phenotypic level might also be distinct. We first tested whether apoptosis contributes to LthFUdR toxicity in C. elegans. We found that loss-of-function mutation of the apoptosis activator ced-4(n1162) and gain-of- function mutation of the apoptosis inhibitor ced-9(n1950) enhance toxicity (Fig. 5a), arguing against apoptosis mediating Lth-FUdR toxicity in C. elegans.

Having used a $C$. elegans-mutant approach to determine that apoptotic mechanisms do not mediate Lth-FUdR toxicity in $C$. elegans, we moved to a targeted RNAi screening approach to 
identify host pathways mediating Lth-FUdR and SE-FUdR toxicity. We performed three- and four-way C. elegans RNAi screens of an RNAi sublibrary composed of 361 C. elegans genes two steps away from pyrimidine, purine, and serine uptake, synthesis, metabolism, or secretion, built based on a reconciled model of C. elegans metabolism we are currently refining (Joshi et al., unpublished). We further added 26 DNA repair, autophagy, and detox pathway genes previously reported to modulate the toxicity of fluoropyrimidines or related compounds ${ }^{14,22}$ (gene list in Supplementary Data 1). To perform RNAi screening using the HB101 background, we developed and validated an RNAicompetent derivative of HB101 that we named EORB1 (Supplementary Fig. 8). Using EORB1, we screened the 387gene RNAi sublibrary in five conditions: (1) no additives, (2) LthFUdR, (3) serine, (4) subLth-FUdR, and (5) SE-FUdR.

We will first describe the results and characterization of the hits of the three-way Lth-FUdR C. elegans RNAi screen. SenGupta et al. $^{22}$ and Scott et al. ${ }^{14}$ demonstrated that 5-FU activates autophagy in C. elegans, and that death requires the autophagyrelated genes bec-1 (C. elegans ortholog of BECLIN 1) and atg-7 (E1-like enzyme involved in conjugation of the ubiquitin-like proteins LGG-1 and ATG-12 to autophagic membranes) ${ }^{14,22}$. In accordance with these reports, our three-way RNAi screen identified four autophagy genes as suppressors of Lth-FUdR toxicity in C. elegans (Supplementary Table 3 and Fig. 5b, c). To better define the role of autophagy, we used 3 approaches to assess the levels of autophagy in worms treated with Lth-FUdR. First, we assessed transcriptional levels of autophagy genes whose expression correlates well with levels of autophagic flux in $C$. elegans $^{23}$, and found increased expression of atg-16.2, atg-18, and bec-1 in worms treated with Lth-FUdR (Supplementary Fig. 9a). Second, we assessed autophagy at the protein level. The most cited approaches to measure autophagy in C. elegans are the measurement of the number of LGG-1::GFP punctae in in vivo imaging analyses, and using a-GFP antibodies to measure LGG$1:: \mathrm{GFP}$ in western blotting assays ${ }^{24}$. However, in isolation, these approaches could be misleading as LGG-1 is subject to autophagic degradation and thus an increased LGG-1::GFP signal could indicate either increased autophagy initiation (increased flux) or decreased lysosomal turnover (decreased flux). Thus, to better assess autophagic flux in C. elegans, we developed and immunopurified antibodies against LGG-1. We validated the antibodies using $\operatorname{lgg}-1 \mathrm{RNAi}$ and LGG-1 overexpression worms (Supplementary Fig. 9b). We then measured autophagic flux in worms by exposing them to the relevant treatments \pm the lysosomal inhibitor chloroquine (CQ). Because CQ blocks lysosomal turnover, the magnitude of the difference in LGG-1 signal between plus and minus CQ reflects the relative level of autophagic flux in any particular condition and can then be compared between conditions (a.k.a. $\Delta \Delta$ LGG-1; see methods for additional details on calculations). Using this metric, we observed 1.5-2 fold increases in autophagic flux in the Lth-FUdR condition (Fig. 5d). Third, as our toxicity readout is embryonic lethality, we assessed autophagic flux in the embryo. For this, we used a previously reported LGG-1 transgenic line ${ }^{25}$ in combination with CQ. In this reporter strain, LGG-1 is fused to a $\mathrm{pH}$-sensitive GFP. Hence, unless lysosomal acidification is perturbed, the GFP signal corresponds to non-acidic autophagosomes (AP). By contrast, in animals treated with an agent that alkalinizes the lysosome (i.e. $\mathrm{CQ}$ ), the GFP signal corresponds to AP + autolysosomes (AL); hence, the ratio $\mathrm{GFP}_{\mathrm{CQ}(+)} / \mathrm{GFP}_{\mathrm{CQ}(-)}=\Delta \mathrm{LGG}-1$ for a given treatment or control. The simplest interpretations of this readout follow: (1) basal autophagic flux: whichever $\Delta$ LGG-1 is observed in wild-type unperturbed animals; (2) reduced or blocked autophagic flux: $\Delta$ LGG-1 is smaller (statistically significant) than $\Delta$ LGG-1 in the control; and (3) increased autophagic flux: $\Delta$ LGG-
1 is larger (statistically significant) than $\Delta \mathrm{LGG}-1$ in the control. Using this metric, we found a $\Delta$ LGG-1 of $~ 50 \%$ in mock and $\sim 300 \%$ in Lth-FUdR (Fig. 5e, f), suggesting Lth-FUdR strongly increases autophagic flux. Altogether, Lth-FUdR promotes high levels of autophagy, and 4 different autophagy genes mediate death in the Lth-FUdR condition. Death not only concurrent, but also dependent on autophagy is the definition of autophagic cell death (ACD). Hence, we propose that worms treated with lethal doses of FUdR are dying through ACD.

In line with ACD mediating Lth-FUdR toxicity in C. elegans, we found that RNAi against aak-2 suppresses toxicity (Fig. $5 \mathrm{~g}$ ). $a a k-2$ encodes for the catalytic subunit of AMP-activated protein kinase (AMPK), a central energy homeostasis kinase that promotes the activation of autophagy ${ }^{26}$ and has been functionally linked to $\mathrm{ACD}^{27}$. AMPK responds to several stresses including DNA damage ${ }^{28}$. Among our RNAi screen hits, we found two DNA repair/damage-related enzymes, MSH-6 and UNG-1 (Fig. 5g). The mismatch-repair enzyme MSH-6 has been shown to mediate 5 -FU toxicity in C. elegans ${ }^{14,22}$. By contrast, ung-1 has not been previously shown to mediate fluoropyrimidine toxicity in C. elegans. UNG-1 is a DNA repair enzyme that catalyzes the removal of uracil misincorporated in DNA. However, if it enters a futile lesion/repair cycle, as when an excess of FdUTP is available to be incorporated into DNA ${ }^{29,30}$ then it promotes DNA damage. Hence, we hypothesized that UNG-1 and AMPK would be part of an axis that activates lethal levels of autophagy in response to FUdR. In support of this hypothesis, we found that RNAi against ung-1 and aak-2 suppresses the activation of autophagy otherwise observed in animals treated with lethal doses of FUdR (Fig. 5h, i). Another RNAi hit, pus-1 (Fig. 5g), provides additional insight into how Lth-FUdR toxicity would be executed in C. elegans. From yeast to mammals pseudouridine synthase (PUS-1) converts uridines present in several RNA classes into pseudouridines $^{31,32}$, and pseudouridylation is required for proper maturation and stability of $\mathrm{RNAs}^{33}$. However, when uracil is fluorinated PUS-1 is irreversibly linked to $i^{34,35}$, reducing the pool of functional RNAs and promoting toxicity ${ }^{36}$. We then tested whether PUS-1 dysfunction would also be upstream of ACD. Indeed, we found that pus-1 RNAi suppresses the hyperactivation of autophagy (Fig. 5h, i). Altogether the data show that UNG-1, AMPK, and PUS-1 are upstream of autophagy in the pathway that promotes death in animals treated with a lethal dose of FUdR. Further, that the suppressors of embryonic lethality also suppress the increased autophagic flux, reinforces the notion that ACD executes death in the Lth-FUdR condition.

In addition to being functionally dysregulated by fluoropyrimidines, mammalian UNG-1 and PUS-1 share a mitochondrial subcellular localization ${ }^{37,38}$. This was intriguing because mitochondrial lipids are emerging as key upstream players in nonapoptotic cell death ${ }^{39-41}$, and, in this sense, the Lth-FUdR suppressor pld-1 is particularly informative because its mammalian homolog, PLD1, produces a lipid signal that activates autophagy ${ }^{42}$. We therefore hypothesized that lipid signals might link mitochondrial dysfunction caused by Lth-FUdR to the activation of lethal levels of autophagy. In support of this hypothesis, we found that ipla-2, T28F3.5, C03H5.4, T09B9.3, and pld-1 not only suppress Lth-FUdR toxicity (Fig. 6a, b) but they also suppress enhanced autophagy (Fig. 6c, d), in line with a model in which lipid signals link mitochondrial dysfunction to ACD.

We then embarked on defining what it is that Lth-FUdR does to the mitochondria. A previous study, found that cytochrome $C$ (cytC) abundance is a good predictor of activation of lethal autophagy downstream of loss of mitochondrial membrane integrity ${ }^{43}$. However, in the context of Lth-FUdR, cytC levels do not correlate with toxicity (Supplementary Fig. 9c). This result 
a

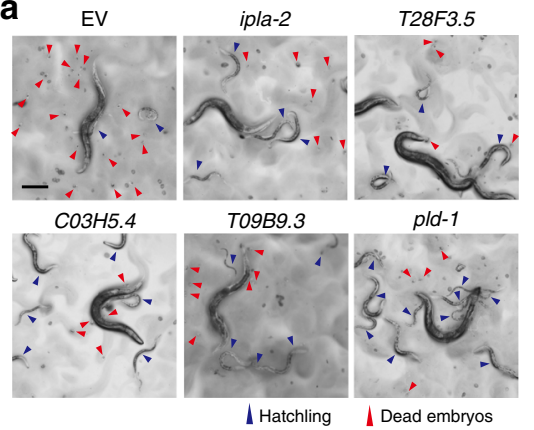

C
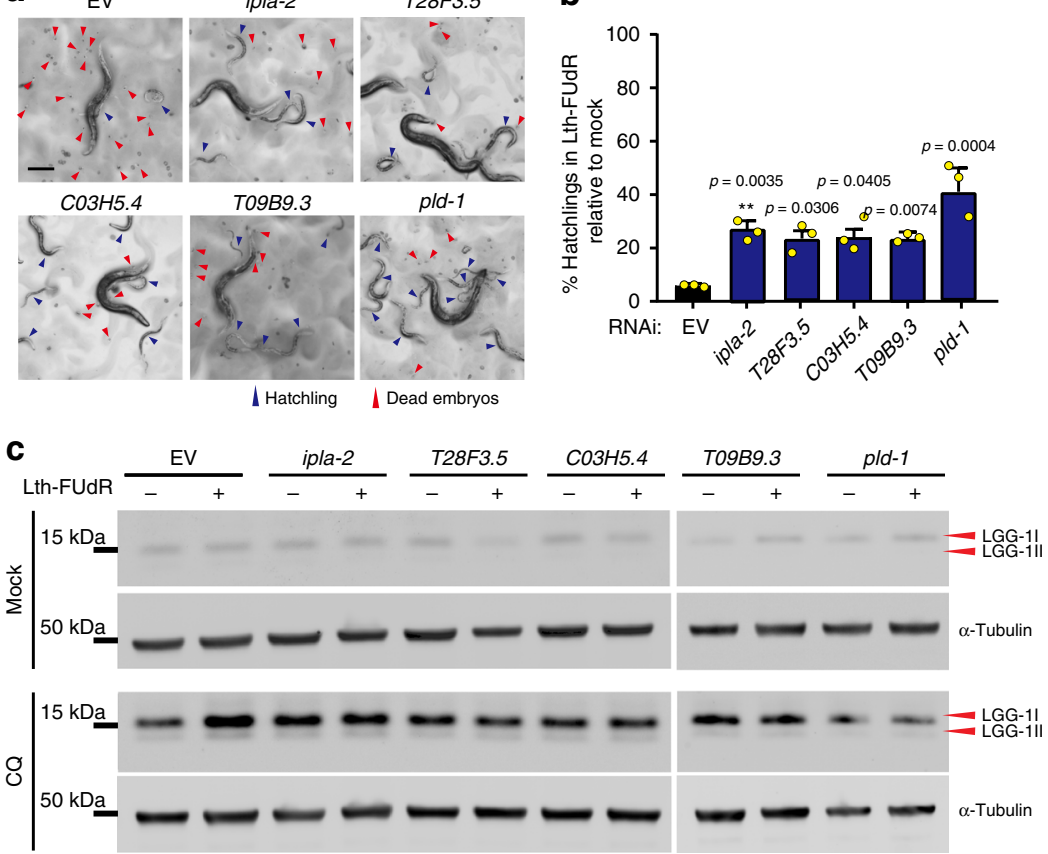

$\frac{\text { СозH5.4 }}{-+} \frac{\text { то9в9.3 }}{-} \frac{p l d-1}{-\quad+}$

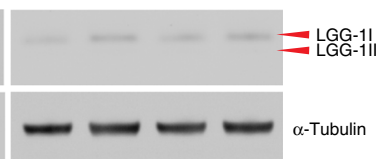

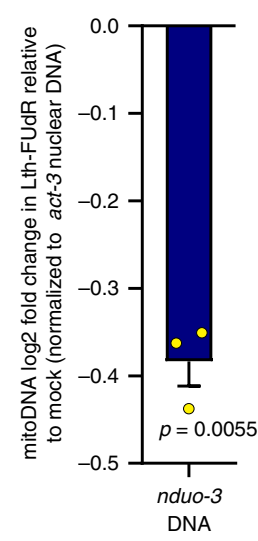

f

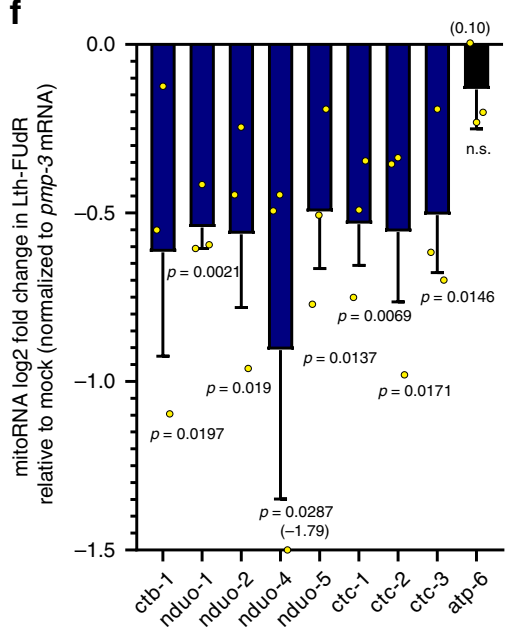

d

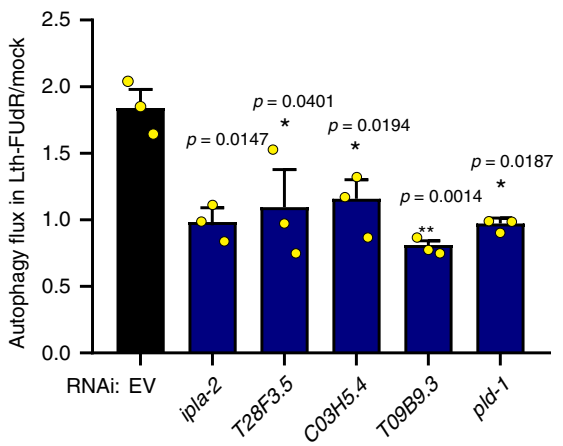

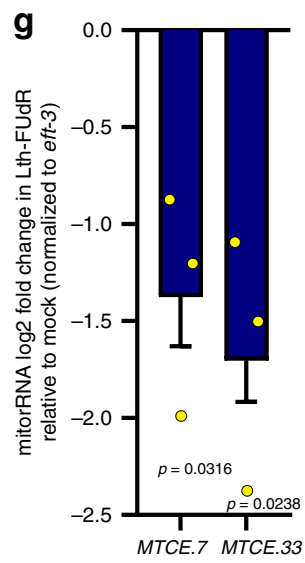

Fig. 6 Autophagy activation in Lth-FUdR depends on mitochondrial lipid metabolism. Throughout this figure: \% hatchlings was analyzed as described in Fig. 1. Statistical significance for \%hatchlings quantification was assessed via two-tailed unpaired nonparametric $t$-test. Statistical significance for western blotting ratio and qPCR fold change was assessed via one-tailed ratio $t$-test. Data are presented as mean values \pm SEM, scale bars $=200 \mu$ m. $n=\#$ independent biological replicates. Source data are provided as a Source Data file. a Representative images of progeny viability of $C$. elegans exposed to LthFUdR $(7.5 \mu \mathrm{g} / \mathrm{mL})$ while cultured on EORB1 EV or RNAi-targeting mitochondrial genes. $n=3$. b Quantification of treatments represented in $\mathbf{a}$. $n=3$. c Representative $\alpha$ LGG-1 western blotting analysis of worms exposed to \pm Lth-FUdR $(7.5 \mu \mathrm{g} / \mathrm{mL}) \pm 8 \mathrm{~h}$ of 20 mM chloroquine while cultured on EORB1 EV or RNAi-targeting mitochondrial genes. Data acquisition as described in Methods. d Quantification of autophagy flux of treatments represented in c. Autophagy flux estimation and interpretation as described in Fig. 5h-I, main text, and Methods. $n=3$. e qPCR analysis of mitochondrial DNA content (nduo-3) relative to nuclear DNA (act-3) in Lth-FUdR worms relative to mock. $n=3$. $\mathbf{f}$ qRT-PCR analysis of the expression/stability of mitochondrially encoded mRNAs relative to the nuclearly encoded mRNA pmp-3 in worms treated with Lth-FUdR relative to mock. $n=3$. $\mathbf{g}$ qRT-PCR analysis of mitochondrially encoded rRNAs normalized to eft-3 (as previously described) in worms treated with Lth-FUdR relative to mock. $n=3$.

suggests that loss of mitochondrial membrane integrity is one of several possible insults to the mitochondria that can trigger lethal autophagy, but it is unlikely to be the one triggering it in animals treated with FUdR. In addition, mitochondrial leakage is the most established trigger of apoptosis ${ }^{44}$. Hence, the cytC negative result is in line with apoptosis not being a mediator of Lth-FUdR toxicity (Fig. 5a). We then searched for other insults that may promote the activation of autophagy in animals treated with FUdR. We found no changes in the mitochondrial oxidative stress response as measured by gst-4 expression (Supplementary Fig. 9d), or the mtUPR response as measured by $h s p-6 \mathrm{mRNA}$ (Supplementary Fig. 9d) and HSP60 protein levels (Supplementary Fig. 9c). However, we did find reduced levels of mitochondrial DNA (Fig. 6e) and mitochondrially encoded mRNAs (Fig. 6f) and rRNA (Fig. 6g) in worms treated with a lethal dose of FUdR. These results align well with PUS-1 and UNG-1 mediating Lth-FUdR toxicity because in mammals futile activation of mitochondrial UNG-1 and malfunction of 
mitochondrial PUS-1 leads to mitochondrial DNA and RNA toxicity, and mitochondrial dysfunction in vitro and in vivo 38,45 . Therefore, although future studies are warranted to fully dissect the mechanisms executing death in animals treated with lethal doses of FUdR, the data presented here fit a model in which FUdR derivatives (likely FUTP and FdUTP) would be incorporated into the host mitochondrial RNAs and DNA, impairing mitochondrial RNA maturation (via PUS-1 inhibition), and promoting mito DNA damage (via futile UNG-1 activity). In turn, AMPK and lipid signals would transduce mitochondrial damage to the cytosol to activate lethal levels of autophagy.
Now, we will describe the results and characterization of the hits of the four-way SE-FUdR C. elegans RNAi screen. As the major toxicity mechanism in the SE-FUdR condition is the classic inhibition of TS, and thymidine-less death has been linked to apoptosis, we first tested whether apoptosis was contributing to SE-FUdR toxicity in C. elegans. However, we found the apoptosis mutants ced-4(n1162) and ced-9(n1950) to further enhance SEFUdR toxicity (Fig. 7a), arguing against apoptosis mediating toxicity in this condition. We then moved onto perform four-way RNAi screening for C. elegans genes mediating SE-FUdR toxicity. The screen hits revealed that the host response to SE-FUdR is a

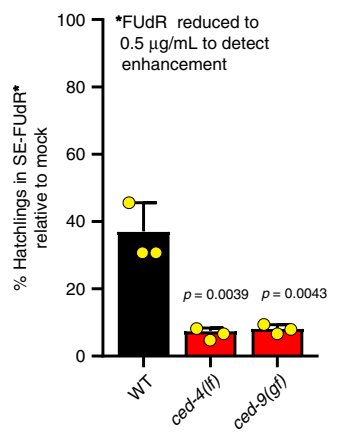

b Lth SE

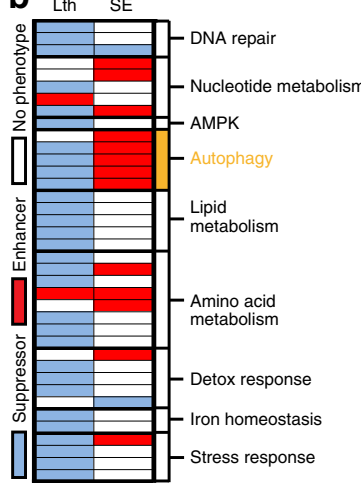

C

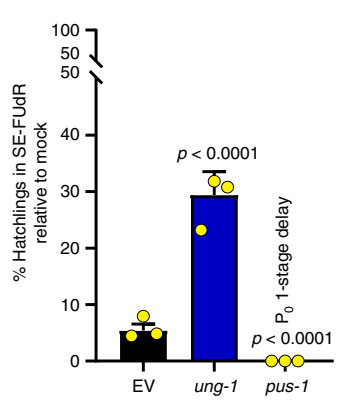

d

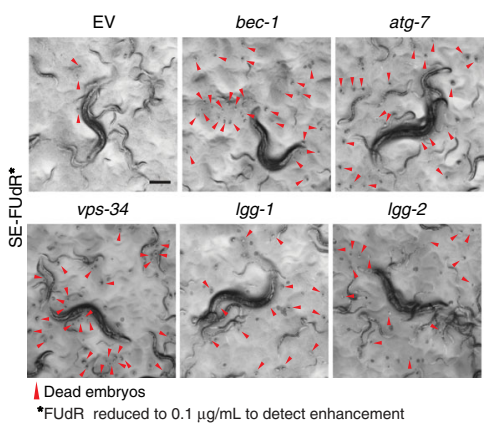

e

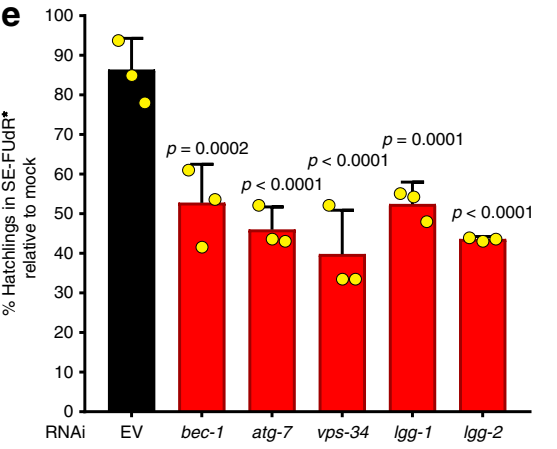

g

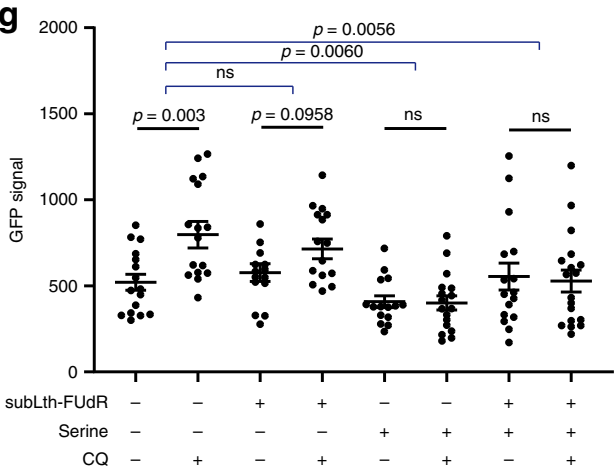

i
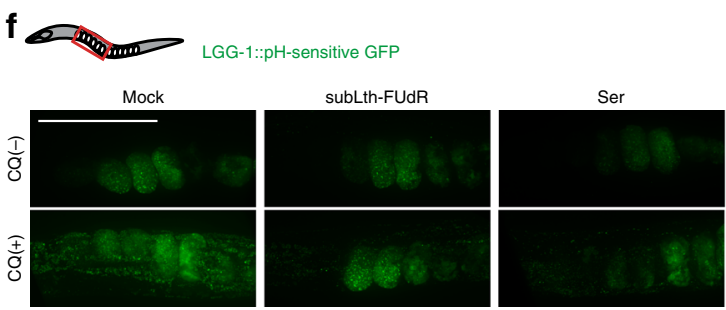

SE-FUdR

\section{h}
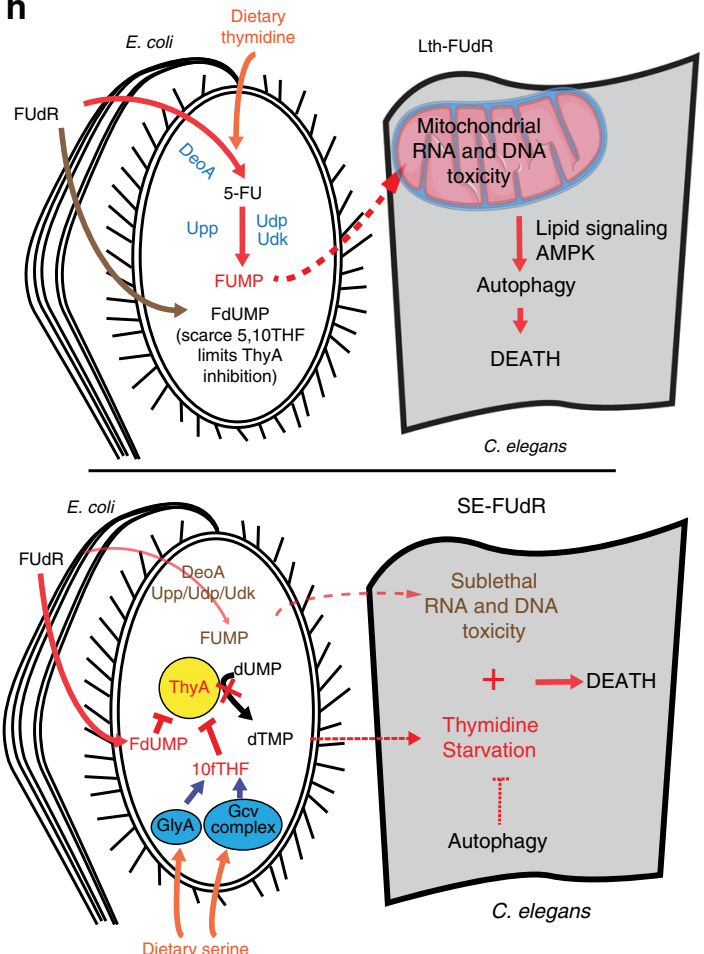

SE-FUdR

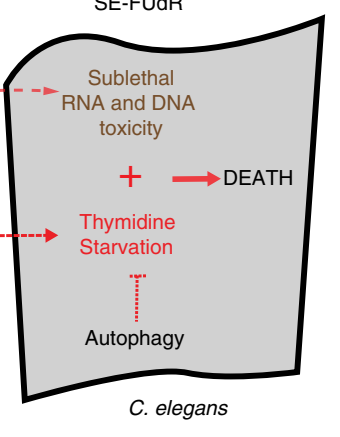

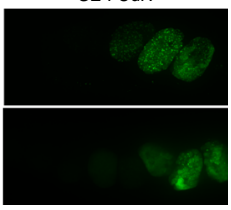

\section{Lth-FUdR SE-FUdR}

\begin{tabular}{rc|c} 
Bacterially driven & Yes & Yes \\
\hline Driven by bacterial secretome & Yes & No \\
\hline E. colis upp, udk dependent & Yes & No \\
\hline Higher toxicity = more FUMP & Yes & No \\
\hline Effect of $E$. coli tdk KO & Enhances & Suppresses \\
\hline Effect of $E$. coli thyA KO & Enhances & None \\
\hline Lowers E. coli \& host Thy & No & Yes \\
\hline Thy (dietary supplementation) & Potentiates & Rescues \\
\hline Targets host mito DNA \& RNA & Yes & No \\
\hline Role host RNA pseudouridylation & Pro-death & Pro-survival \\
\hline Role host autophagy & Pro-death & Pro-survival \\
\hline
\end{tabular}


Fig. 7 Host response to Lth-FUdR and SE-FUdR are distinct. Throughout this figure: \% hatchlings was analyzed as described in Fig. 1. Statistical significance was assessed via two-tailed unpaired nonparametric $t$-test for $\%$ hatchlings quantification. Data are presented as mean values \pm SEM. $n=\#$ independent biological replicates. Source data are provided as a Source Data file. a Quantification of progeny viability of WT, ced-4(n1162), and ced-9 (n1950) mutant C. elegans cultured on EORB1 lawns treated with $0.5 \mu \mathrm{g} / \mathrm{mL}$ plus $1.5 \mathrm{mg} / \mathrm{mL}$ serine (lower dose of FUdR used to enable detection of SEFUdR enhancers). $n=3$. b GO distribution of the three-way (left) and four-way (right) hits from the C. elegans RNAi screen for modulators of Lth-FUdR and SE-FUdR, respectively. Enriched functional class (Fisher's exact test $p<0.005$ ) denoted yellow. c Quantification of progeny viability of $C$. elegans exposed to SE-FUdR $(1 \mu \mathrm{g} / \mathrm{mL}$ FUdR) while cultured on EORB1 EV or RNAi against ung-1 or pus-1. $n=3$. d Representative images of progeny viability of $C$. elegans cultured on EORB1 EV or autophagy RNAi lawns treated with $0.1 \mu \mathrm{g} / \mathrm{mL}$ plus $1.5 \mathrm{mg} / \mathrm{mL}$ serine (lower dose of FUdR used to enable detection of enhancers). Scale bar $=200 \mu \mathrm{m} . n=3$. e Quantification of treatments represented in $\mathbf{d} . n=3$. $\mathbf{f}$ In vivo imaging of embryos expressing LGG-1::GFP(pHsensitive) treated in EORB1 lawns with $1 \mu \mathrm{g} / \mathrm{mL} \mathrm{FUdR,} 1.5 \mathrm{mg} / \mathrm{mL}$ serine, and FUdR plus serine, $\pm 6 \mathrm{~h}$ on $20 \mathrm{mM}$ chloroquine. Scale bar $=100 \mu \mathrm{m}$. $n=3$. $\mathbf{g}$ Quantification of GFP signal of treatments represented in $\mathbf{f}$. Analysis as described in Fig. $5 f$. $\mathbf{h}$ Working model of the host response to Lth-FUdR and SEFUdR. In the Lth-FUdR condition, some derivatives of FUMP generated in the worm (e.g. FUTP) misincorporate into mitochondrial RNAs preventing RNA maturation and function, whereas others (e.g. FdUTP) are incorporated into DNA, promoting detrimental levels of DNA repair. Then lipid signals and AMPK link the consequent mitochondrial dysfunction to the activation of autophagic cell death. In the SE-FUdR condition, mito RNAs and DNA are not major targets. Instead, C. elegans die of dTMP deficiency, and its consequent thymidine-less death, which autophagy can alleviate. i List of distinctive characteristics of Lth-FUdR and SE-FUdR.

remarkably distinct from the response to Lth-FUdR. From the nine genes that were hits in both screens, only two show the same phenotype in both conditions (Fig. $7 \mathrm{~b}$ and Supplementary Table 3). One of these genes is ung-1, which suppresses LthFUdR (Fig. 5g) and SE-FUdR (Fig. 7c) toxicity. In contrast, the other seven genes that are hits in both screens show opposite phenotypes. pus-1 suppresses Lth-FUdR toxicity (Fig. 5g) and enhances SE-FUdR toxicity (Fig. 7c), which is in line with a more prevalent role for RNA toxicity in the Lth-FUdR than in the SEFUdR condition. Most striking, the autophagy genes, as a class, have opposite phenotypes in the two screens. While autophagy mediates Lth-FUdR toxicity, RNAi against the autophagy genes bec-1, atg-7, $\operatorname{lgg}-1, \operatorname{lgg}$-2, and $v p s-34$ further enhances SE-FUdR toxicity (Fig. $7 \mathrm{~d}, \mathrm{e}$ ), suggesting that autophagy promotes death downstream of fluororibonucleotide toxicity, but protects from death during thymidine starvation. One autophagy gene, atg-7, acts distinctively as its inactivation does not suppress Lth-FUdR toxicity but enhances SE-FUdR toxicity. However, autophagy independent from ATG-7 (a.k.a. non-conventional autophagy) has been reported ${ }^{46,47}$, and ATG-7 modulates the DNA damageresponsive tumor suppressor and cell-death mediator $\mathrm{p} 53^{48}$. Therefore, the protective role of ATG-7 in SE-FUdR toxicity may occur through mechanisms distinct from autophagy. We then measured the levels of autophagy in the SE-FUdR toxicity condition. We found no changes in the levels of expression of autophagy genes (Supplementary Fig. 9e) or autophagic flux by western blots of gravid adults (Supplementary Fig. 9f, g). However, when exposed to serine, embryos show similar GFP signal in the absence and presence of CQ (Fig. 7f, g), suggestive of reduced autophagic flux. Altogether, although several aspects of the death mechanisms remain to be elucidated, it is clear that LthFUdR and SE-FUdR are distinctively executed in the host. Further supporting this notion, we observe no changes in mitochondrial DNA (Supplementary Fig. 9h) or RNA content (Supplementary Fig. 9i) in the SE-FUdR condition, and, correspondingly, AMPK and the lipid metabolism genes that suppress Lth-FUdR toxicity do not suppress SE-FUdR toxicity (Fig. 7b). Altogether, the results show that dietary serine not only changes metabolic flux in E. coli, and with that the level of toxicity of FUdR, but also redefines the host response to FUdR toxicity (working model in Fig. 7h).

\section{Discussion}

Diet and microbiota are attractive targets for therapeutic intervention. However, the dominance of correlative and in vitro studies on the effects that diet and microbiota have on the host response to drugs has limited the development of therapeutic interventions targeting diet, microbiota, or both. Here, we used a tractable system that enables molecular dissection of four-way diet, drug, microbe, and host interactions in vivo. With this fourway model system, we dissected the microbe and host response to FUdR, and how they both change when serine is supplemented to the diet.

We first show that Lth-FUdR toxicity is bacterially driven. Although thymidine-less death is the best characterized mechanism of FUdR toxicity, E. coli-mediated thymidine starvation is not how E. coli promotes Lth-FUdR toxicity in our experimental setup. However, when serine is supplemented to the diet, this changes. Dietary serine enables the inhibition of E. coli's thymidylate synthase (TS), reducing the dTMP pool in E. coli and consequently in C. elegans. Together, the data presented here suggest that the precursors for the synthesis of 5,10-mTHF, an essential TS cofactor, are limiting in our experimental setup. More importantly, the data show that single dietary changes can shift the microbe's metabolism and, consequently, the host response to a drug to the point of transforming a non-lethal dose into a lethal one. In practical terms, the fact that supplementation with a single dietary metabolite can greatly enhance the potency of $E$. coli-mediated FUdR toxicity as well as shift its mechanism of action emphasizes the need to employ standardized media conditions when studying drug mechanisms in model organisms. Indeed, in C. elegans research, peptone concentrations from different commercial providers are not standardized. Thus, some results garnered from $C$. elegans studies using drugs supplemented to the media may be influenced by the varied nutrient compositions of media and the consequent distinct interactions with microbial and host metabolism. More broadly, the mechanisms of action of dietary thymidine and serine show that the microbiota can affect the efficacy or toxicity of drugs through at least two mechanisms: (1) Directly, via metabolizing the drug (i.e. increased conversion of FUdR into FUMP; thymidine mechanism). This mechanism has been exploited to uncover microbiota-drug interactions ${ }^{49}$; and (2) Indirectly, via converting dietary nutrients into metabolites that in turn change the microbe's capabilities to alter the host response to the drug (i.e. $E$. coli-mediated conversion of dietary serine into 5,10-mTHF enabling thymidine-less death in E. coli and hence in the worm; serine mechanism). To the best of our knowledge, this mechanism is demonstrated here for the first time.

The significance of using simplified tractable models of microbe-host co-metabolism resides in unveiling the complexity of the molecular interactions that may affect drug treatment outcomes, and serving as guide for mechanistic studies in higher organisms. At first sight, C. elegans may seem too unique to 
inform host-microbiota interactions in higher organisms. Bacteria serve as microbiota and food source in C. elegans ${ }^{50}$. Hence, bacteria are the principal source of micro and macronutrients, and this may seem different from mammals. However, the mammalian gut microbiota has a critical role in providing essential nutrients and in digesting the complex carbohydrates, proteins, and fats that reach the lower gastrointestinal tract in mammals $^{51}$. Furthermore, bacterial lysis, and the consequent release of cell content, is part of the normal mammalian gut dynamics $^{52}$. The enterohepatic system permits exchange of metabolites, byproducts, and xenobiotics between the intraluminal intestine, the bloodstream, and animal tissues ${ }^{53}$. Indeed, microbiota-derived nucleosides and nucleotides can be found in blood and other host organs in mammals ${ }^{54-56}$ including humans ${ }^{57}$, suggesting that microbial nucleotide metabolism could impact host nucleotide metabolism and hence the metabolism of chemotherapeutics beyond C. elegans. Similarly, studies in mammals show bacterially converted dietary folates, and bacterially derived serine in host tissues ${ }^{9,10,12}$. It is also notable that panels of probiotic bacteria that include $E$. coli can differentially activate chemotherapeutics in vitro ${ }^{58,59}$. Therefore, although only suggestive, the current mammalian evidence is in line with diet being capable of modulating fluoropyrimidine efficacy and toxicity through altering the metabolism of gut microbes in the clinical setting. Therefore, microbes could account, at least in part, for the variability in fluoropyrimidine responsiveness that cannot be explained by the genetics of the patient or the tumor ${ }^{60}$. Most important, and exemplifying the value of simplified model systems, the notion introduced here that microbe-derived "natural" metabolites can have a significant impact on the efficacy and toxicity of drugs is relevant on its own, because even the most detailed studies to date base the screens for microbial activities modulating drug efficacy or toxicity on biochemical searches for microbe-derived drug derivatives (degradation products or modified versions of the administered drug) ${ }^{49}$. Our work reveals a limitation of these drug-derivative screens, as they would miss microbiota activities (i.e. conversion of dietary serine into 5,10mTHF) capable of, for example, transforming a non-lethal dose of FUdR into a lethal one.

Notwithstanding, the most surprising finding from this work is that dietary serine also alters, and in cases reverses, the role that host pathways have in the response to FUdR. Examples include RNA modification (pus-1) and autophagy (bec-1, $\operatorname{lgg}$ - 1 , $\lg g$-2, and $v p s$-34) executing death in the Lth-FUdR condition and protecting from death in the SE-FUdR condition. Furthermore, even for genes having similar roles in both conditions, the underlying mechanisms may be distinct. For instance, ung- 1 is the only gene with a suppressor phenotype in both conditions. Nevertheless, based on the mitochondrial DNA results, it is likely that UNG-1's toxic role is due to a futile cycle of removal and reincorporation of fluorouracil in the mitochondrial DNA of FUdR-treated animals. However, in the SE-FUdR condition, mitochondrial DNA is not depleted and fluororibonucleotide toxicity is not the main mechanism of E. coli-driven toxicity. Instead, the combination of low levels of dTTP and relative high levels of dUTP and FdUTP would favor the incorporation of fluorinated and non-fluorinated uracils in genomic DNA as previously reported ${ }^{30,61}$. Hence, it is likely that in the SE-FUdR condition, UNG-1 is toxic because it enters a futile cycle of removal and reincorporation of uracil into the genomic DNA. Altogether, even when a surface-level interpretation of the outcome (100\% embryonic lethality) would lead one to believe that the same mechanisms underlie death in these two conditions-dead embryos look grossly identical, worms and bacteria are isogenic, and the drug is the same-the underlying mechanisms in the microbe and the host in the presence or absence of dietary supplementation with serine are distinct to the point that the same molecular players have opposite roles (Fig. 7i). Although our study identifies these striking subphenotypic distinctions, it leaves many questions unanswered. Future studies would be necessary to fully dissect the underlying death mechanisms in both the Lth-FUdR and SE-FUdR conditions. This will likely be a challenging endeavor, as the mechanisms by which cells die of thymidine-less death have remained unknown for decades ${ }^{61,62}$. Nevertheless, the understanding that distinct mechanisms can underlie the same treatment outcomes should guide future research; in particular, it should encourage limiting the use of correlative studies for translational purposes.

Humans host more than 1500 species in the gut, and the composition varies between and within individuals ${ }^{63}$. Each of these microbes can distinctly metabolize dietary components and drugs. The dietary nutrient and drug derivatives from each microbe can be further metabolized or alter the physiology of other microbes and the host, building chains of events alternatively or simultaneously triggered by dietary, drug, microbe and host metabolites, byproducts, and signaling molecules. Hence, we can speculate that the complexity of drug-microbe-host cometabolism in vivo is astronomical. Therefore, the complexity of the simplified four-way interactions presented here highlight both the extensive need for mechanistic studies, and the challenges we face to realize the full therapeutic potential of the microbiota.

\section{Methods}

C. elegans and E. coli strains. C. elegans strains N2 (Bristol, UK), MT2547 (ced-4 mutant n1162) and MT4770 (ced-9 mutant n1950) were obtained from the Caenorhabditis Genetics Center (CGC). MAH2 $15^{25}$ is a kind gift from Dr. Malene Hansen. Unless otherwise noted, experiments were initiated with synchronized L1 larvae obtained by egg bleaching and overnight synchronization in S-buffer. Wildtype E. coli strain BW25113 and Keio KO strains were obtained from the E. coli Genetic Stock Center. E. coli strain HB101 was obtained from CGC. EORB1 and the EORB1 RNAi library were constructed in our lab.

E. coli culturing and compound supplementation. For every biological replicate fresh E. coli streaks or library stamps on LB-carbenicilin $50 \mu \mathrm{g} / \mathrm{mL}$ (RNAi clones) or LB-kanamycin $25 \mu \mathrm{g} / \mathrm{mL}$ (Keio KO library) were used. Bacterial cultures were started from single colonies or using a sterilized inoculating hedgehog, and grown overnight for 14-16 h. Keio clones were grown overnight in LB-kanamycin $15 \mu \mathrm{g} /$ $\mathrm{mL}, \mathrm{RNAi}$ clones were grown overnight in LB-carbenicilin $50 \mu \mathrm{g} / \mathrm{mL}$ in the absence of IPTG (or any other additives). The parental strain BW25113 was grown in plain LB. For aeration, flasks were shaken at $250 \mathrm{rpm}$, and $1.2 \mathrm{~mL}$ deep 96 -well plates at $1000 \mathrm{rpm}$. For targeted experiments, bacteria were harvested by centrifugation at room temperature and resuspended to $\mathrm{OD}_{600 \mathrm{~nm}}=20$ in S-buffer $(\sim 20 \times$ concentrated). For screening, $1.2 \mathrm{~mL}$ bacterial cultures were resuspended with $20 \mu \mathrm{L}$ of S-buffer. Concentrated E. coli were seeded onto NGM or NGM-RNAi plates immediately and never exposed to the cold.

For dietary supplementation, metabolites were dissolved in water (unless otherwise stated), filter-sterilized, seeded on NGM or RNAi plates, and dried in biosafety hood. Concentrated bacteria were seeded as soon as metabolites dried out FUdR was dissolved in water to $100 \times$ concentration, filter-sterilized, and added directly onto bacterial lawns immediately after lawns were dried. Seeding dietary supplement, fresh bacteria, and FUdR in that order, and adding supplements and FUdR within a $2 \mathrm{~h}$ window of seeding fresh bacteria is critical to observe the full effect of the supplements. Synchronized hatchlings were seeded the same day for all experiments except for C. elegans RNAi experiments ( $24 \mathrm{~h}$ later to activate RNAi). When post-developmental transfer (i.e. embryogenic competence in Suppl. Figure 1e) was necessary, worms were grown in the E. coli background in which they were later tested.

Imaging and image analysis. Percent hatchling was measured by taking $\geq 5$ images of each treatment or mock plate per biological replicate, and at least three independent biological replicates were carried out for all assays. Images were taken on Zeiss Axio Zoom.v16 dissecting microscope, PlanNeoFluar Z ×2.3/0.57 FWD objective, zoom $\times 30$. Hatchlings, live and dead eggs and adults were quantitated assisted by ImageJ object counting tool. Values in figures are presented as "\% hatchling relative to mock", meaning the number of hatchings was first normalized to total progeny (hatchlings + live embryos + dead embryos) in each treatment and then normalized to the \% hatchlings in the corresponding non-FUdR (mock) treatment. This provides a quantitative measurement controlling for other variables such as the time of scoring. For Keio clones and RNAi experiments, treatments are normalized first to mock of the same Keio clone or RNAi and then to WT, which takes into account the potential effect of the Keio or RNAi clones on worm health 
However, we did not observe Keio or RNAi only effects in any of the E. coli or worm genes inactivations reported as hits.

Estimation example: if BW25113 $+1 \mu \mathrm{g} / \mathrm{mL}$ FUdR $=21$ hatchlings $/ 185$ progenies (hatchlings + live embryos + dead embryos), and BW25113 untreated $=$ 197 hatchlings/201 progenies, this implies \% hatchlings for BW25113 in FUdR relative to untreated is $11.58 \%(11.35 / 98 \times 100)$. Then, if $d e o A$ shows hatchling/ progeny ratios of 168/176 in FUdR and 194/199 in control, by the same calculation deoA \% hatchling is $97.91 \%$; thus, if reproducible, $d e o A$ is a suppressor.

For enhancers, lower doses of FUdR are used and the calculations take into account the effects of FUdR relative to wild type, but in this case the WT + FUdR will show subtle toxicity. As an example, if BW25113 $+0.5 \mu \mathrm{g} / \mathrm{mL} \mathrm{FUdR}=80$ hatchlings/152 progenies and BW25113 untreated $=198$ hatchlings $/ 200$ progenies, $\%$ hatchlings for WT BW25113 in this condition is $53.16 \%$. If $n d k+0.5 \mu \mathrm{g} / \mathrm{mL}$ FUdR has 25 hatchlings/ 148 progenies and $n d k$ untreated has 205 hatchlings/208 progenies, \% hatchlings in worms fed $n d k$ is $17.13 \%$, so $n d k$ is an enhancer because when cultured on this E. coli mutant worms produce less viable progeny than when fed wild-type E. coli.

All toxicity measurements were repeated $\geq 3$ times and the mean \pm SEM are presented.

Occasionally, embryogenic competence was calculated to identify enhancers of toxicity. The calculation is inclusive of hatchlings, live eggs and dead eggs produced per worm, and is influenced by the effect of FUdR ( \pm supplements) on both the rate of development and the fertility of the $\mathrm{P}_{0}$ s. Therefore, lesser embryogenic competence or $\mathrm{P}_{0}$ developmental delay compared to wild-type or unsupplemented reveals enhancers, whereas increased embryogenic competence reveals suppressors. Specifically, 10 worms were singly transferred to test plates, and allowed to lay progeny for $24 \mathrm{~h}$. Next day the total number of progeny (live + dead embryos) were counted per plate. Normalization of embryogenic competence was done as described above for \%hatchlings.

Dietary metabolite four-way screen. Amino acids were freshly dissolved to 10 $\mathrm{mg} / \mathrm{mL}$ in water (except tyrosine: $1 \mathrm{mg} / \mathrm{mL}$ ), aluminum foiled, rocked for $12 \mathrm{~h}$ at RT, and filter-sterilized. Seven 1:2 serial dilutions were made and amino acids were seeded to the appropriate concentrations into 96-well plates with $100 \mu \mathrm{L}$ NGM per well. Once dry, wells were seeded with $8 \mu \mathrm{L}$ of fresh $20 \times \mathrm{HB101}$. Once dry, 2 plates (duplicate) were seeded with $5 \mu \mathrm{L}$ of $250 \mu \mathrm{g} / \mathrm{mL}$ FUdR (final $12.5 \mu \mathrm{g} / \mathrm{mL}$ ). The remaining two plates (duplicate) were left as no-FUdR controls to test the potential toxicity of the amino acids. Once dry, 25 synchronized hatchlings were seeded per well and incubated at $20^{\circ} \mathrm{C}$. Altogether, the following conditions were tested: (1) Negative control: $12.5 \mu \mathrm{g} / \mathrm{mL}$ FUdR-only, which leads to $100 \%$ sterile adults but no developmental delay; (2) Positive control: $12.5 \mu \mathrm{g} / \mathrm{mL}$ FUdR supplemented with $5 \mathrm{mg} / \mathrm{mL}$ thymidine, which leads to $100 \%$ larval arrest; (3) Amino acid toxicity control: wells supplemented only with the 8 doses of amino acids (but no-FUdR), to test for the potential toxicity of the amino acids; and (4) Screening wells: wells supplemented with the 8 doses of amino acids and $12.5 \mu \mathrm{g} / \mathrm{mL}$ FUdR. After 60 and $72 \mathrm{~h}$, wells were scored as follows for worm developmental stages: $1=\mathrm{L} 1 / \mathrm{dead}$, $2=\mathrm{L} 2$ larvae, $3=\mathrm{L} 3$ larvae, $4=\mathrm{L} 4$ larvae, $5=$ Young adults $(<5$ eggs in body), $6=$ Gravid adults ( $>5$ eggs in body). Only wells which showed a $\geq 1$ stage delay in FUdR + amino acid compared to FUdR-only, and the AA showed no toxicity on its own, were considered hits.

Supernatant and pellet test. Saturated overnight $E$. coli cultures were reinoculated 1:50 in liquid NGM (Nematode Growth Media without agar), and grown to $\mathrm{OD}_{600 \mathrm{~nm}} \sim 1$, at which point water (mock), Lth-FUdR $(50 \mu \mathrm{g} / \mathrm{mL})$, or subLth-FUdR $(1 \mu \mathrm{g} / \mathrm{mL}) \pm$ thymidine $(5 \mathrm{mg} / \mathrm{mL})$ or serine $(1.5 \mathrm{mg} / \mathrm{mL})$ were added. After 2 more hours of incubation at $37^{\circ} \mathrm{C}$, bacteria were pelleted and resuspended in $\geq 50$ volumes of water three times to remove residual FUdR from the bacterial suspension. On the final wash, $\mathrm{OD}_{600 \mathrm{~nm}}$ absorbance was measured and bacteria were resuspended to $\mathrm{OD}_{600 \mathrm{~nm}}=20$, and $100 \mu \mathrm{L}$ were seeded onto NGM plates. Once lawns were dried, 100 hatchlings were seeded and incubated at $25^{\circ} \mathrm{C}$. At multiple times between 60 and $96 \mathrm{~h}$ plates were imaged for scoring of developmental stages and fertility.

The same setup was used for supernatant experiments, but after centrifugation the supernatants were taken on ice, filtered-sterilized through a $0.22-\mu \mathrm{m}$ filter, lyophilized overnight, and resuspend in water to make 1 and $5 \times$ concentrated supernatants. $100 \mu \mathrm{L}$ of the sterile supernatant resuspensions were seeded on top of upp; $u d k ; u d p$ triple KO E. coli lawns. We used 3KO lawns to avoid in-plate conversion of FUdR remaining in the supernatant. Once supernatants dried, 100 hatchlings were seeded and incubated at $25^{\circ} \mathrm{C}$. At multiple times between 60 and $96 \mathrm{~h}$, plates were imaged for scoring of developmental stages and fertility.

E. coli four-way screen. Keio screen for mediators of SE-FUdR toxicity was performed at $25^{\circ} \mathrm{C}$ in 8 conditions: (1) mock (water), (2) FUdR $0.25 \mu \mathrm{g} / \mathrm{mL}$, (3) serine $1.5 \mathrm{mg} / \mathrm{mL}$, and $(4-8)$ serine $1.5 \mathrm{mg} / \mathrm{mL}$ plus FUdR from 0.05 to $0.25 \mu \mathrm{g} / \mathrm{mL}$ (setup depicted in Fig. 3a). Keio clones were grown overnight in $1.2 \mathrm{~mL}$ of LBkanamycin in deep 96 -well plates at $37^{\circ} \mathrm{C}$ and $1000 \mathrm{rpm}$. Cultures were pelleted, supernatants discarded, and pellets resuspended in $20 \mu \mathrm{L}$ of S-buffer. Eight microliters of bacterial suspension were seeded into wells containing $100 \mu \mathrm{L}$ of NGM plus or minus serine. Once bacteria dried, $5 \mu \mathrm{L}$ of $1,2,3,4$, or $5 \mu \mathrm{g} / \mathrm{mL}$ FUdR were seeded onto bacteria lawns (final doses $0.05,0.1,0.15,0.2$, and $0.25 \mu \mathrm{g} / \mathrm{mL}$, respectively). Once dried, 50 synchronized hatchlings were seeded and incubated at $25^{\circ} \mathrm{C}$. The non-FUdR controls allowed us to determine whether any given E. coli KO clone would adversely affect development or fertility on their own or in combination with serine only. FUdR-only wells were scored after $60-72 \mathrm{~h}$ relative to WT E. coli BW25113 as follows: $-2=$ no hatchlings, $-1=$ fewer hatchlings, $0=$ similar to WT control, $1=$ more hatchlings than control, $2=$ similar to noFUdR control. FUdR + serine wells were scored after $60-96 \mathrm{~h}$ for developmental delay or embryogenic competence relative to WT E. coli BW25113 control as follows: $-2=$ severely delayed P0, $-1=$ moderate delay P0/few eggs laid, $0=$ similar to control (sterile adult), $1=$ some hatchlings, $2=$ similar to no-serine control. Only genes showing a suppressor or enhancer phenotype in the FUdR plus serine condition at multiple doses or in $>2$ screen repeats were considered hits. All Keio hits were verified by PCR and sequencing. Primary hits are presented in Supplementary Table 2 as: blue $=$ suppressor of toxicity; orange $=$ enhancer of toxicity. Light blue or orange, represents phenotype observed in only 1 of screen 3 repeats. Hits belonging to overrepresented metabolic pathways were retested in $6 \mathrm{~cm}$ NGM plates and quantitated for \% hatchlings in sublethal FUdR $(0.25 \mu \mathrm{g} / \mathrm{mL})$ \pm serine $(1.5 \mathrm{mg} / \mathrm{mL})$, and the results are presented in main figures. Primary screen hits that were not retested in $6 \mathrm{~cm}$ plates are depicted as NRT in Supplementary Table 2. Retested and verified hits and non-hits are marked as " ل", and retested but not validated primary hits (phenotype did not repeat) are marked as "X".

Bacterial growth in plate. E. coli BW25113 and HB101 were cultured and seeded in NGM \pm additives plates as normally done for Lth-FUdR or SE-FUdR tests. After $48 \mathrm{~h}$ exposure to treatments, cells were recovered from the plates, resuspended in equal volumes, and biomass $\left(\mathrm{OD}_{600 \mathrm{~nm}}\right)$ and viability ( $\mathrm{CFU}$ of serial dilutions from $10^{0}$ to $10^{-7}$ ) were quantitated.

Bacterial growth in liquid. HB101, BW25113, and Keio hits were hedgehog seeded from frozen stocks onto LB agar omnitray plates. Next day, $100 \mu \mathrm{L}$ of liquid NGM \pm subLth-FUdR \pm serine were stamp-seeded in duplicate. Absorbance at $600 \mathrm{~nm}$ was recorded longitudinally using a SpectraMax plate reader maintained at $37^{\circ} \mathrm{C}$ in a continuous shaking mode. Measurements were independently carried out more than three times.

Bacteria and C. elegans metabolomics. For Lth-FUdR and dietary supplementation-related metabolomics, single colonies of E. coli BW25113 or HB101 were used to inoculate $500 \mathrm{~mL}$ of LB and incubated overnight for $14 \mathrm{~h}$ at $37^{\circ} \mathrm{C} 250 \mathrm{rpm}$. Bacteria were harvested by centrifugation, and resuspended in $25 \mathrm{~mL}$ of S-buffer. Concentrated bacteria were seeded on nylon membranes placed on the surface of the NGM plates to avoid "contamination" of the bacteria with NGM agar media. For this, $5 \mathrm{~mL}$ of concentrated bacterial suspension were dried onto $90 \mathrm{~mm}$ Nylon membranes (VWR 7402-009) by vacuum filtration in a sterile porcelain Buchner funnel. The nylon membranes loaded with bacteria were placed on the surface of $15 \mathrm{~cm}$ NGM agar plates with or without the respective supplementations (i.e. $\pm \mathrm{FUdR}$ and \pm thymidine or \pm serine). After $24 \mathrm{~h}$ at $25^{\circ} \mathrm{C}$, E. coli were harvested by washing the bacteria off the membrane with $50 \mathrm{~mL}$ of cold liquid NGM. Bacteria were then washed two more times with $50 \mathrm{~mL}$ of cold water. To confirm effectiveness of the treatments, $100 \mu \mathrm{L}$ of bacterial suspension were seeded on NGM plates without any additives, hatchlings were seeded on these lawns, and incubated for $60 \mathrm{~h}$ at $25^{\circ} \mathrm{C}$; a time at which they were scored for progeny viability. The remaining of the washed bacterial pellets were flash frozen, lyophilized, and kept at $-80^{\circ} \mathrm{C}$ for later extraction as described below. Samples verified via parallel hatchling-viability controls were processed for LC-MS analyses.

For supernatant analyses of thymidine-enhanced toxicity, single colonies of $E$ coli BW25113 were used to inoculate $20 \mathrm{~mL}$ of LB broth and incubated overnight for $14 \mathrm{~h}$ at $37^{\circ} \mathrm{C} 250 \mathrm{rpm}$. Next morning cultures were pelleted, washed, and resuspended in equal volume of liquid NGM. Ten milliliters of this bacterial resuspension were used to inoculate $500 \mathrm{~mL}$ of liquid NGM supplemented with mock, or subLth-FUdR \pm thymidine $(5 \mathrm{mg} / \mathrm{mL})$, and incubated for another $2 \mathrm{~h}$ at $37^{\circ} \mathrm{C} 250 \mathrm{rpm}$. Mock and treated cultures were then harvested by centrifugation. The supernatants were filter-sterilized to remove residual bacteria. Aliquots (10 $\mathrm{mL}$ ) were spiked in with the internal standards listed below, frozen, lyophilized, and reconstituted right before LC-MS in 1:1 acetonitrile: water. The bacterial pellets were washed two more times with $50 \mathrm{~mL}$ of cold water, flash frozen and lyophilized, and kept at $-80^{\circ} \mathrm{C}$ for later extraction as described below.

To generate worm metabolomics samples, 50,000 synchronized 1-day gravid adult worms were harvested, washed in a $40 \mu \mathrm{m}$ mesh $1 \times$ with $50 \mathrm{~mL}$ of NGM, incubated for $5 \mathrm{~min}$ in clean media to allow gut clearance, and then mesh-washed again with $50 \mathrm{~mL}$ cold liquid NGM and $1 \times$ with $50 \mathrm{~mL}$ of cold water, and immediately frozen in liquid nitrogen and lyophilized, and kept at $-80^{\circ} \mathrm{C}$ for later extraction as described below.

Right before resuspending the lyophilates, a master mix of internal standards was prepared by mixing the following compounds at a final concentration of $20 \mathrm{ng} /$ $\mu \mathrm{L}$ in HPLC-grade methanol: (1) 1,3-15N2 Uracil (Cambridge Isotope lab NLM637-PK); (2) Uridine-13C9,15N2 5'-triphosphate (Sigma \#645672); and (3) Glycine-13C2, (Sigma \#283827). Then, the spike-in control master mix was diluted 1:50 in $80 \%$ methanol. $20 \mathrm{mg}$ of lyophilized bacteria or $5 \mathrm{mg}$ of lyophilized worms 
were resuspended in $500 \mu \mathrm{L}$ of the diluted internal standard solution. Samples were mixed with $200 \mu \mathrm{L}$ of $100 \mu \mathrm{m}$ silica beads and disrupted $5 \times$ for $30 \mathrm{~s}$ in a minibeadeater ${ }^{\mathrm{TM}}-8$ disruptor with cooling on ice for $2 \mathrm{~min}$ after each cycle. Extracts were cleared through two rounds of $15 \mathrm{~min}$ centrifugation at $4^{\circ} \mathrm{C}$ and $20,000 \times g$, and then lyophilized. To measure endogenous metabolites, samples were reconstituted right before LC-MS in 1:1 acetonitrile: water. Samples were separated on a Luna aminopropyl column $(3 \mu \mathrm{m}, 150 \mathrm{~mm} \times 1.0 \mathrm{~mm}$ I.D., Phenomenex $)$ or a CORTECS T3 column $(2.7 \mu \mathrm{m}, 150 \mathrm{~mm} \times 2.1 \mathrm{~mm}$ I.D., Waters $)$ and analyzed using an Agilent 6530 Q-TOF, an Agilent 6540 Q-TOF, or a ThermoScientific Q Exactive Plus. The Luna column was used in negative mode with the following buffers and linear gradient: $\mathrm{A}=95 \%$ water, $5 \%$ acetonitrile $(\mathrm{ACN}), 10 \mathrm{mM}$ ammonium hydroxide, $10 \mathrm{mM}$ ammonium acetate; $\mathrm{B}=95 \% \mathrm{ACN}, 5 \%$ water; $100 \%$ to $0 \% \mathrm{~B}$ from 0 to $30 \mathrm{~min}$ and $0 \% \mathrm{~B}$ from 30 to $40 \mathrm{~min}$; flow rate $50 \mu \mathrm{L} / \mathrm{min}$. The $\mathrm{T} 3$ column was used in positive mode with the following buffer and linear gradient: $\mathrm{A}=95 \%$ water, $5 \% \mathrm{ACN}, 10 \mathrm{mM}$ ammonium acetate, $0.1 \%$ formic acid; $\mathrm{B}=95 \% \mathrm{ACN}, 5 \%$ water; $0 \%$ to $100 \%$ B from 0 to $30 \mathrm{~min}$ and $100 \%$ B from 30 to $40 \mathrm{~min}$; flow rate $200 \mu \mathrm{L} / \mathrm{min}$. The identity of each metabolite was confirmed by comparing retention times to standard compounds and tandem MS data with the METLIN metabolite database.

To measure fluorometabolites, samples were reconstituted right before LC-MS in 2:1:1 water:methanol:acetonitrile, and $3 \mu \mathrm{L}$ was further analyzed by liquid chromatography-mass spectrometry (LC-MS) as follows. Metabolite profiling was performed using Ultimate 3000 UHPLC (Dionex) coupled to Q Exactive Plus-Mass spectrometer (QE-MS, ThermoScientific). A hydrophilic interaction chromatography method (HILIC) employing an Xbridge amide column $(100 \times$ $2.1 \mathrm{~mm}$ i.d., $3.5 \mu \mathrm{m}$; Waters) was used for polar metabolite separation. The LC method is modified from a previous study ${ }^{64}$ : The HPLC (Ultimate 3000 UHPLC) is coupled to QE-MS (ThermoScientific) for metabolite separation and detection. An Xbridge amide column $(100 \times 2.1 \mathrm{~mm}$ i.d., $3.5 \mu \mathrm{m}$; Waters $)$ is employed for compound separation at room temperature. The mobile phase A is $5 \mathrm{mM}$ ammonium acetate and in water, $\mathrm{pH} 6.8$, and mobile phase $\mathrm{B}$ is acetonitrile. The linear gradient used is as follows: $0 \mathrm{~min}, 85 \% \mathrm{~B} ; 1.5 \mathrm{~min}, 85 \% \mathrm{~B}, 5.5 \mathrm{~min}, 35 \%$ B; $10 \mathrm{~min}, 35 \% \mathrm{~B}, 10.5 \mathrm{~min}, 35 \% \mathrm{~B}, 14.5 \mathrm{~min}, 35 \% \mathrm{~B}, 15 \mathrm{~min}, 85 \% \mathrm{~B}$, and $20 \mathrm{~min}$, $85 \% \mathrm{~B}$. The flow rate was $0.15 \mathrm{~mL} / \mathrm{min}$ from 0 to $10 \mathrm{~min}$ and 15 to $20 \mathrm{~min}$ and $0.3 \mathrm{~mL} / \mathrm{min}$ from 10.5 to $14.5 \mathrm{~min}$. The QE-MS is equipped with a HESI probe with related parameters set as below: heater temperature, $120^{\circ} \mathrm{C}$; sheath gas, 30 ; auxiliary gas, 10; sweep gas, 3 ; spray voltage, $3.0 \mathrm{kV}$ for the positive mode and $2.5 \mathrm{kV}$ for the negative mode; capillary temperature, $320^{\circ} \mathrm{C}$; S-lens, 55 ; scan range $(\mathrm{m} / \mathrm{z}): 70$ to 900 for pos mode (1.31 to $12.5 \mathrm{~min})$ and neg mode (1.31 to $6.6 \mathrm{~min}$ ) and 100 to 1000 for neg mode (6.61 to $12.5 \mathrm{~min}$ ); resolution: 70,000; automated gain control (AGC), $3 \times 10^{6}$ ions. Customized mass calibration was performed before data acquisition. LC-MS peak extraction and integration were performed using commercially available software Sieve 2.2 (ThermoScientific).

For all metabolites, peak sizes of the target metabolites $(\mathrm{P})$ were normalized to the corresponding internal standard (IS) peak area (amino acids to Glycine-13C2, nucleotides to UMP-13C9,15N2, and nucleosides to Uracil-15N2). The normalized peak value from the treatments was then normalized to the mock control from the same biological replicate. Therefore, target metabolite relative abundance was estimated as follows $(\mathrm{P} / \mathrm{IS})_{\text {treatment }} /(\mathrm{P} / \mathrm{IS})_{\text {mock }}$ from 3 to 5 independent biological replicates

EORB1 strain construction. To construct the RNAi-competent EORB1 strain, E. coli HB101 was first transiently made $r e c A+$. Then $r n c 14$ was interrupted with mini-Tn10 transposon introduced via P1 transduction. Transduced colonies were selected in $25 \mu \mathrm{g} / \mathrm{mL}$ tetracycline (Tet). Tet-resistant colonies were picked and restreaked for two rounds, and then cured of the recA plasmid by growing in LB at $44^{\circ} \mathrm{C}$. Streaking onto LB plates containing chloramphenicol $(25 \mu \mathrm{g} / \mathrm{mL})$ and treating with UV confirmed loss of chloramphenicol resistance and the reappearance of sensitivity to UV. Loss of RNAseIII function should prevent the maturation of rRNAs. The rnc-phenotype was confirmed as accumulation of the 30S rRNA precursor (Supplementary Fig. 8a). T7 polymerase (under the control of the LacUV5 promoter) and the transcriptional repressor LacI were then introduced via lysogenization ( $\lambda \mathrm{DE} 3$ Lysogenization Kit; Novagen 69734). The presence of inducible T7 polymerase was confirmed by western blot (Supplementary Fig. 8b). The final EORB1 strain has the genotype [HB101], rnc-, lacI-lacUV5p-T7. EORB1 was confirmed to be competent for feeding RNAi by phenotypic analysis. RNAi against the genes $d a f-2$, unc-22, $d p y-13$, and pos-1 displayed the expected phenotypes when delivered via feeding RNAi from EORB1 (Supplementary Fig. 8c-f). To construct the EORB1 library, we miniprepped (QIAprep 96-plus Miniprep Kit; Qiagen 27291) the screened constructs from HT115 Ahringer RNAi library. EORB1 was made chemically competent by $\mathrm{CaCl}_{2}$ preparation. $5 \mu \mathrm{L}$ of the plasmid minipreps were added to $50 \mu \mathrm{L}$ of chemically competent EORB1 heat shocked at $42^{\circ} \mathrm{C}$, and grown overnight in liquid LB-carbenicilin $50 \mu \mathrm{g} / \mathrm{mL}$. Transformants underwent a second and third round of selection on solid and liquid LB-carb50, after which glycerol stocks were made.

Four-way C. elegans RNAi screen and verification. RNAi screens were performed at $25^{\circ} \mathrm{C}$ in 96-well plates. RNAi clones were grown in $1.2 \mathrm{~mL}$ of LB carbenicillin for $12-16 \mathrm{~h}$ in 96 -deep-well plates at $37^{\circ} \mathrm{C}$ and $1000 \mathrm{rpm}$. Cultures were harvested by centrifugation. Pellets were suspended in residual LB volume, and
$8 \mu \mathrm{L}$ were seeded into wells containing $100 \mu \mathrm{L}$ of $\mathrm{NGM}$ with $50 \mu \mathrm{g} / \mathrm{mL}$ carbenicillin and $5 \mathrm{mM}$ IPTG (though $1 \mathrm{mM}$ IPTG is sufficient). Wells were then seeded with $\pm 2.5 \mu \mathrm{g} / \mathrm{mL}$ FUdR, and $\pm 5 \mathrm{mg} / \mathrm{mL}$ thymidine or $\pm 1.5 \mathrm{mg} / \mathrm{mL}$ serine as appropriate After drying in biosafety hood, plates were left overnight at room temperature to allow RNAi induction. The next day, $25 \mathrm{~L} 1$ larvae were seeded and grown for 60-72 h. At this point, controls were confirmed to have 0 hatchlings and plates were scored as follows: $0=$ similar to control, $1=\sim 1-10$ hatchlings, $2=\sim 11-50$ hatchlings, $3=\sim 51-100$ hatchlings, $4=>100$ hatchlings, and $-1=$ developmental delay. All RNAi hits were sequence verified. Primary hits are presented in Supplementary Table 3 as: blue $=$ suppressor of toxicity; orange $=$ enhancer of toxicity and white $=$ no different from WT control. Light blue or orange, represents phenotype observed in only 1 of screen 3 repeats. Hits belonging to overrepresented pathways were retested and quantitated for $\%$ hatchlings in $6 \mathrm{~cm}$ plates, and the results are presented in main figures. Not retested hits are depicted as NRT in Supplementary Table 3, whereas retested and verified hits and non-hits are marked as " "ل, and retested RNAi clones in which the phenotype did not repeat are marked as " $\mathrm{X}$ ".

LGG1 antibody. Anti-LGG1 (Rabbit) antibodies were generated by Covalab (Villeurbanne, France) against peptides FEKRRAEGDKIRRKY and GQLYQDHHEEDLFLY (sequence optimized and kindly shared by Vincent Galy). Serum was immunopurified and anti-LGG-1 specificity was validated in western blots using WT, $\operatorname{lgg}-1$ RNAi, and $\operatorname{lgg}-1$ OE samples as controls (Supplementary Fig. 9b).

Western blotting. For all western blots to measure autophagy levels, samples were prepared from two plates of 2000 worms grown at $25^{\circ} \mathrm{C}$ for each treatment. After $50 \mathrm{~h}, 2000$ worms from one of plates were harvested and reseeded in NGM plates with the same additives (i.e. \pm subLth-FUdR and \pm serine) plus $20 \mathrm{mM}$ chloroquine (CQ). After $8 \mathrm{~h}$ of treatment, worms were harvested from both sets of plates $( \pm \mathrm{CQ})$ washed $3 \mathrm{X}$ with S-buffer to remove residual bacteria, and immediately frozen in liquid nitrogen. Frozen worm pellets were resuspended in 2 volumes of $1 \times$ RIPA buffer (Sigma \#R0278) and sonicated. Aliquots of soluble proteins were quantitated using BCA Thermo kit (Pierce 23227), and the rest mixed with $3 \times$ SDS-PAGE sample loading buffer, and incubated for $5 \mathrm{~min}$ at $85^{\circ} \mathrm{C}$. $30 \mu \mathrm{g}$ of protein were loaded to each lane of a 4-12\% Bis-tris gel (Fisher NP0322BOX), and ran for $55 \mathrm{~min}$ at $200 \mathrm{~V}$ in MES running buffer. Gels were transferred to $0.2-\mu \mathrm{m}$ nitrocellulose membranes in transfer buffer (Fisher NP0006) with $20 \%$ methanol at $30 \mathrm{~V}$ for $45 \mathrm{~min}$. The membranes were stained with Ponceau red to evaluate the quality of the SDS-PAGE and transfer, and then blocked with Intercept ${ }^{\circledast}$ (PBS) Blocking Buffer (Li-Cor 927-70001) with 0.1\% Tween for $4 \mathrm{~h}$. Membranes were exposed to primary antibodies including $\alpha$-LGG1 1:250 (custom made by Covalab), anti- $\alpha$ tubulin 1:10,000 (DSHB 4A1), $\alpha$-HSP60 1:1000 (DSHB HSP60), $\alpha$-cytC 1:1000 (Abcam 37BA11) overnight at $4{ }^{\circ} \mathrm{C}$. Membranes were washed $3 \times 15 \mathrm{~min}$ with PBST $(0.1 \%$ Tween) and incubated with secondary antibodies $1: 10,000$ for $1 \mathrm{~h}$ at room temperature (IRDye ${ }^{\circledast}$ 800CW Goat anti-Mouse IgG Secondary Antibody, CAT\#925-32210, and IRDye ${ }^{\circledast}$ 800CW Goat anti-Rabbit IgG Secondary Antibody, CAT\#925-32211), and imaged by Li-Cor Odyssey imager. For cytC western blots, samples were prepared from a single plate and processed similarly to a previous protocol with the modifications described above ${ }^{43}$.

Autophagy flux in treated animals relative to mock was calculated by measuring integrated density $(I)$ of each band and normalized as shown in Fig. 5h. Autophagy flux relative to mock was then estimated as: $\Delta \Delta \mathrm{LGG}-1=\left(\left(\mathrm{LGG}-1_{\text {treatment }+\mathrm{CQ}} /\right.\right.$ Tubulin $\left._{\text {treatment }+\mathrm{CQ}}\right) /\left(\mathrm{LGG}-1_{\text {treatment no CQ }} /\right.$ Tubulin $\left.\left._{\text {treatment no } \mathrm{CQ}}\right)\right) /\left(\left(\mathrm{LGG}-1_{\text {mock }+\mathrm{CQ}} /\right.\right.$ Tubulin $\left._{\text {mock }+\mathrm{CQ}}\right) /\left(\mathrm{LGG}-1_{\text {mock no } \mathrm{CQ}} /\right.$ Tubulin $\left.\left._{\text {mock no } \mathrm{CQ}}\right)\right)$.

In vivo fluorescent reporter-based measurement of autophagic flux. Two hundred 1-day-old adult worms (strain MAH215) from each treatment (mock, sublth-FUdR, serine, sublth-FUdR + serine, or Lth-FUdR) were harvested with Sbuffer, washed $3 \times$, and incubated $30 \mathrm{~s}$ with egg-prep bleaching solution. Worms were then washed $3 \times$ in S-buffer, and mounted onto agar pad on glass slides (Thermo, 3011) with \#1.5 coverslip (Fisher $1.522 \times 22 \mathrm{~mm}$ ).

$Z$-stacks to measure autophagic flux in embryos were captured on Nikon Eclipse Ti spinning disc confocal microscope, $40 \times / 1.3 \mathrm{NA}$ objective, $500 \mathrm{~ms}$ exposure time and $80 \%$ laser intensity. All the images from each biological replicate were identically processed using ImageJ. First, maximum projections of the fluorescent and bright field channels were created in ImageJ. Then the embryos were cropped out for analysis. ImageJ plot profiling combined with thresholding was used to detect and quantitate LGG-1::GFP dots. Average GFP signal of $>15$ individuals from $\geq 4$ biological replicates are depicted for each treatment. Then, $\triangle \mathrm{LGG}-1=($ average GFP signal in $\mathrm{CQ}(+)) /($ average GFP signal in $\mathrm{CQ}(-))$ is calculated for each treatment (i.e. Lth-FUdR) and control. Lastly, all repeats $(n=4)$ of $\Delta \mathrm{LGG}-1_{\text {treatment }}$ and $\Delta \mathrm{LGG}-1_{\text {control }}$ are compared using ratio t-test.

qPCR analysis. One-day gravid adults were harvested, washed in a $40 \mu \mathrm{m}$ nylon mesh, and quickly frozen in liquid nitrogen. Samples were kept at $-80^{\circ} \mathrm{C}$ until RNA extraction. Total RNA was isolated using TriReagent (MRC). To quantitate expression of mitochondrially encoded RNAs (no introns), DNA was removed by DNAse I (Sigma AMPD1) treatment prior to retrotranscription with random hexamer primers. All qRT-PCR reactions were performed in triplicate. Median \pm 
SEM of $d d \mathrm{Ct}$ is reported ${ }^{65}$. For measurement of mitochondria to nuclear DNA ratios, 100 gravid worms from mock and treatments were lysed with Worm Lysis Buffer $(50 \mathrm{mM} \mathrm{KCl} ; 10 \mathrm{mM}$ Tris pH 8.3; $2.5 \mathrm{mM} \mathrm{MgCl} 2 ; 0.45 \% \mathrm{NP}-40 ; 0.45 \%$ Tween-20; $0.01 \%$ gelatin) with $0.3 \%$ proteinase $\mathrm{K}$ and the supernatants containing DNA were collected and used as template. iTaq Universal SYBR Green Supermix (Biorad 1725120) reactions run in BioRad CFX96 thermocycler were analyzed using $d d \mathrm{Ct}^{65}$.

Statistics and data representation. All statistical analyses were performed in Graphpad Prism. Outliers were detected and removed from analyses using the ROUT method. For \%hatchlings, embryogenic competency, and GFP intensity quantifications, unpaired nonparametric $t$-test was used to make single comparisons between a specific treatment and mock control in. Ratio $t$-test was used to compare all ratios including $\mathrm{qPCR}$ fold changes, $\triangle \mathrm{LGG}-1, \mathrm{CFU}$, and normalized LC-MS ratios. Unless otherwise stated, exact $p$-values are provided within the figures. Unless otherwise stated, data in this study are presented as mean values \pm SEM. All experiments were performed and quantitated at least three independent times.

Reporting summary. Further information on research design is available in the Nature Research Reporting Summary linked to this article.

\section{Data availability}

The source data underlying Figs. 1b-m, 2b-j, 3b, c, 4a-g, 4i, j, 5a-i, 6a-h, 7a-g, Supplementary Figs. 1b-f, 3b, c, 5a-e, 6, 7a-e, 8a-f, 9a-i, and Supplementary Tables 1-3 are provided as a Source Data file. All other data supporting the findings of the study are available from the corresponding authors upon request.

Received: 10 August 2017; Accepted: 15 April 2020;

Published online: 22 May 2020

\section{References}

1. Won, C. S., Oberlies, N. H. \& Paine, M. F. Mechanisms underlying food-drug interactions: Inhibition of intestinal metabolism and transport. Pharmacol. Ther. 136, 186-201 (2012).

2. Ruggiero, A. et al. The role of diet on the clinical pharmacology of oral antineoplastic agents. Eur. J. Clin. Pharmacol. 68, 115-122 (2012).

3. Hitchings, R. \& Kelly, L. Drug metabolism as a community effort. Cell Metab. https://doi.org/10.1016/j.cmet.2019.07.005 (2019).

4. Malet-Martino, M. Clinical studies of three oral prodrugs of 5-fluorouracil (Capecitabine, UFT, S-1): a review. Oncologist https://doi.org/10.1634/ theoncologist.7-4-288 (2002).

5. Ducker, G. S. \& Rabinowitz, J. D. One-carbon metabolism in health and disease. Cell Metabol. https://doi.org/10.1016/j.cmet.2016.08.009 (2017).

6. Snell, K., Natsumeda, Y. \& Weber, G. The modulation of serine metabolism in hepatoma 3924A during different phases of cellular proliferation in culture. Biochem. J. https://doi.org/10.1042/bj2450609 (1987).

7. Ullman, B., Lee, M., Martin, D. W. \& Santi, D. V. Cytotoxicity of 5 fluoro $2^{\prime}$ deoxyuridine: requirement for reduced folate cofactors and antagonism by methotrexate. Proc. Natl Acad. Sci. USA https://doi.org/10.1073/pnas.75.2.980 (1978).

8. Kok, D. E. et al. Bacterial folate biosynthesis and colorectal cancer risk: more than just a gut feeling. Crit. Rev. Food Sci. Nutr. https://doi.org/10.1080/ 10408398.2018.1522499 (2018).

9. Rong, N., Selhub, J., Goldin, B. R. \& Rosenberg, I. H. Bacterially synthesized folate in rat large intestine is incorporated into host tissue folyl polyglutamates. J. Nutr. https://doi.org/10.1093/jn/121.12.1955 (1991)

10. Asrar, F. M. \& O'Connor, D. L. Bacterially synthesized folate and supplemental folic acid are absorbed across the large intestine of piglets. $J$. Nutr. Biochem. https://doi.org/10.1016/j.jnutbio.2005.02.006 (2005).

11. Maynard, C., Cummins, I., Green, J. \& Weinkove, D. A bacterial route for folic acid supplementation. BMC Biol. https://doi.org/10.1186/s12915-018-0534-3 (2018).

12. Nakade, Y. et al. Gut microbiota-derived D-serine protects against acute kidney injury. JCI Insight https://doi.org/10.1172/jci.insight.97957 (2018).

13. García-González, A. P. et al. Bacterial metabolism affects the C. elegans response to cancer chemotherapeutics. Cell 169, 431-441.e8 (2017).

14. Scott, T. A. et al. Host-microbe co-metabolism dictates cancer drug efficacy in C. elegans. Cell 169, 442-456.e18 (2017).

15. Pryor, R. et al. Host-microbe-drug-nutrient screen identifies bacterial effectors of metformin therapy. Cell https://doi.org/10.1016/j.cell.2019.08.003 (2019).

16. Chi, C. et al. Nucleotide levels regulate germline proliferation through modulating GLP-1/Notch signaling in C. elegans. Genes Dev. 30, 307-320 (2016).
17. Locasale, J. W. Serine, glycine and one-carbon units: cancer metabolism in full circle. Nat. Rev. Cancer https://doi.org/10.1038/nrc3557 (2013).

18. Panosyan, E. H. et al. Asparagine depletion potentiates the cytotoxic effect of chemotherapy against brain tumors. Mol. Cancer Res. https://doi.org/10.1158/ 1541-7786.MCR-13-0576 (2014).

19. Tsun, Z. Y. \& Possemato, R. Amino acid management in cancer. Semin. Cell Dev. Biol. https://doi.org/10.1016/j.semcdb.2015.08.002 (2015)

20. Lukey, M. J., Katt, W. P. \& Cerione, R. A. Targeting amino acid metabolism for cancer therapy. Drug Discov. Today https://doi.org/10.1016/j. drudis.2016.12.003 (2017).

21. Steiert, P. S., Stauffer, L. T. \& Stauffer, G. V. The lpd gene product functions as the L protein in the Escherichia coli glycine cleavage enzyme system. $J$. Bacteriol. https://doi.org/10.1128/jb.172.10.6142-6144.1990 (1990).

22. SenGupta, T. et al. Base excision repair AP endonucleases and mismatch repair act together to induce checkpoint-mediated autophagy. Nat. Commun. 4, 2674 (2013).

23. Zhang, H. et al. Guidelines for monitoring autophagy in Caenorhabditis elegans. Autophagy https://doi.org/10.1080/15548627.2014.1003478 (2015).

24. Palmisano, N. J. \& Meléndez, A. Detection of autophagy in Caenorhabditis elegans using GFP::LGG-1 as an autophagy marker. Cold Spring Harb. Protoc. https://doi.org/10.1101/pdb.prot086496 (2016).

25. Chang, J. T., Kumsta, C., Hellman, A. B., Adams, L. M. \& Hansen, M. Spatiotemporal regulation of autophagy during Caenorhabditis elegans aging. Elife https://doi.org/10.7554/eLife.18459 (2017).

26. Kim, J., Kundu, M., Viollet, B. \& Guan, K. L. AMPK and mTOR regulate autophagy through direct phosphorylation of Ulk1. Nat. Cell Biol. https://doi org/10.1038/ncb2152 (2011).

27. Ha, S. et al. Phosphorylation of $\mathrm{p} 62$ by AMP-activated protein kinase mediate autophagic cell death in adult hippocampal neural stem cells. J. Biol. Chem. https://doi.org/10.1074/jbc.M117.780874 (2017).

28. Zhang, D. et al. The interplay between DNA repair and autophagy in cancer therapy. Cancer Biol. Ther. https://doi.org/10.1080/15384047.2015.1046022 (2015).

29. Pettersen, H. S. et al. UNG-initiated base excision repair is the major repair route for 5-fluorouracil in DNA, but 5-fluorouracil cytotoxicity depends mainly on RNA incorporation. Nucleic Acids Res. https://doi.org/10.1093/nar/ gkr563 (2011).

30. Seiple, L., Jaruga, P., Dizdaroglu, M. \& Stivers, J. T. Linking uracil base excision repair and 5-fluorouracil toxicity in yeast. Nucleic Acids Res. https:// doi.org/10.1093/nar/gkj430(2006).

31. Chen, J. \& Patton, J. R. Cloning and characterization of a mammalian pseudouridine synthase. RNA https://doi.org/10.1017/S1355838299981591 (1999).

32. Ansmant, I., Massenet, S., Grosjean, H., Motorin, Y. \& Branlant, C. Identification of the Saccharomyces cerevisiae RNA: pseudouridine synthase responsible for formation of $\psi 2819$ in $21 \mathrm{~S}$ mitochondrial ribosomal RNA. Nucleic Acids Res. https://doi.org/10.1093/nar/28.9.1941 (2000).

33. Spenkuch, F., Motorin, Y. \& Helm, M. Pseudouridine: still mysterious, but never a fake (uridine)! RNA Biol. https://doi.org/10.4161/ 15476286.2014.992278 (2014).

34. Huang, L., Pookanjanatavip, M., Gu, X. \& Santi, D. V. A conserved aspartate of tRNA pseudouridine synthase is essential for activity and a probable nucleophilic catalyst. Biochemistry 37, 344-351 (1998).

35. Gu, X., Liu, Y. \& Santi, D. V. The mechanism of pseudouridine synthase I as deduced from its interaction with 5-fluorouracil-tRNA. Proc. Natl Acad. Sci. USA https://doi.org/10.1073/pnas.96.25.14270 (1999).

36. Zhao, X. \& Yu, Y. T. Incorporation of 5-fluorouracil into U2 snRNA blocks pseudouridylation and pre-mRNA splicing in vivo. Nucleic Acids Res. https:// doi.org/10.1093/nar/gkl1084 (2007).

37. Slupphaug, G. et al. Nuclear and mitochondrial forms of human uracil-DNA glycosylase are encoded by the same gene. Nucleic Acids Res. https://doi.org/ 10.1093/nar/21.11.2579 (1993).

38. Bykhovskaya, Y., Casas, K., Mengesha, E., Inbal, A. \& Fischel-Ghodsian, N. Missense mutation in pseudouridine synthase 1 (PUS1) causes mitochondrial myopathy and sideroblastic anemia (MLASA). Am. J. Hum. Genet. https://doi. org/10.1086/421530 (2004).

39. Magtanong, L., Ko, P. J. \& Dixon, S. J. Emerging roles for lipids in nonapoptotic cell death. Cell Death Differ. 23, 1099-1109 (2016).

40. Nikoletopoulou, V., Markaki, M., Palikaras, K. \& Tavernarakis, N. Crosstalk between apoptosis, necrosis and autophagy. Biochim. Biophys. Acta 1833, 3448-3459 (2013).

41. Dixon, S. J. et al. Human haploid cell genetics reveals roles for lipid metabolism genes in nonapoptotic cell death. ACS Chem. Biol. 10, 1604-1609 (2015).

42. Dall'Armi, C. et al. The phospholipase D1 pathway modulates macroautophagy. Nat. Commun. 1, 142(2010).

43. Zhou, B. et al. Mitochondrial permeability uncouples elevated autophagy and lifespan extension. Cell https://doi.org/10.1016/j.cell.2019.02.013 (2019). 
44. Jeong, S. Y. \& Seol, D. W. The role of mitochondria in apoptosis. J. Biochem. Mol. Biol. https://doi.org/10.5483/bmbrep.2008.41.1.011 (2008).

45. Endres, M. et al. Increased postischemic brain injury in mice deficient in uracilDNA glycosylase. J. Clin. Invest. https://doi.org/10.1172/JCI200420926 (2004).

46. Chang, T.-K. K. et al. Ubal functions in Atg7- and Atg3-independent autophagy. Nat. Cell Biol. 15, 1067-78 (2013).

47. Nishida, Y. et al. Discovery of Atg5/Atg7-independent alternative macroautophagy. Nature 461, 654-658 (2009).

48. Lee, I. H. et al. Atg7 modulates p53 activity to regulate cell cycle and survival during metabolic stress. Science 336, 225-8 (2012).

49. Zimmermann, M., Zimmermann-Kogadeeva, M., Wegmann, R. \& Goodman, A. L. Mapping human microbiome drug metabolism by gut bacteria and their genes. Nature https://doi.org/10.1038/s41586-019-1291-3 (2019).

50. Samuel, B. S., Rowedder, H., Braendle, C., Félix, M. A. \& Ruvkun, G. Caenorhabditis elegans responses to bacteria from its natural habitats. Proc. Natl Acad. Sci. USA https://doi.org/10.1073/pnas.1607183113 (2016).

51. Oliphant, K. \& Allen-Vercoe, E. Macronutrient metabolism by the human gut microbiome: major fermentation by-products and their impact on host health. Microbiome https://doi.org/10.1186/s40168-019-0704-8 (2019).

52. Derrien, M. \& van Hylckama Vlieg, J. E. T. Fate, activity, and impact of ingested bacteria within the human gut microbiota. Trends Microbiol. https:// doi.org/10.1016/j.tim.2015.03.002 (2015).

53. Watkins, J. B. \& Klaassen, C. D. Comprehensive Toxicology 3rd edn, https:// doi.org/10.1016/B978-0-12-801238-3.02122-X (Elsevier, 2017).

54. Fergus, C., Barnes, D., Alqasem, M. A. \& Kelly, V. P. The queuine micronutrient: charting a course from microbe to man. Nutrients https://doi. org/10.3390/nu7042897 (2015)

55. Proietti, M. et al. ATP released by intestinal bacteria limits the generation of protective IgA against enteropathogens. Nat. Commun. https://doi.org/ 10.1038/s41467-018-08156-z (2019).

56. Nakayama, H. et al. Intestinal anaerobic bacteria hydrolyse sorivudine, producing the high blood concentration of 5-(E)-(2-bromovinyl)uracil that increases the level and toxicity of 5-fluorouracil. Pharmacogenetics 7, 35-43 (1997).

57. Poore, G. D. et al. Microbiome analyses of blood and tissues suggest cancer diagnostic approach. Nature https://doi.org/10.1038/s41586-020-2095-1 (2020)

58. Lehouritis, P. et al. Local bacteria affect the efficacy of chemotherapeutic drugs. Sci. Rep. https://doi.org/10.1038/srep14554 (2015).

59. Lehouritis, P., Stanton, M., McCarthy, F. O., Jeavons, M. \& Tangney, M. Activation of multiple chemotherapeutic prodrugs by the natural enzymolome of tumour-localised probiotic bacteria. J. Control. Release https://doi.org/ 10.1016/j.jconrel.2015.11.030 (2016).

60. Scartozzi, M. et al. 5-Fluorouracil pharmacogenomics: Still rocking after all these years? Pharmacogenomics https://doi.org/10.2217/pgs.10.167 (2011).

61. Van Triest, B., Pinedo, H. M., Giaccone, G. \& Peters, G. J. Downstream molecular determinants of response to 5 -fluorouracil and antifolate thymidylate synthase inhibitors. Ann. Oncol. https://doi.org/10.1023/ A:1008351221345 (2000)

62. Khodursky, A., Guzmán, E. C. \& Hanawalt, P. C. Thymineless death lives on: new insights into a classic phenomenon. Annu. Rev. Microbiol. https://doi.org/ 10.1146/annurev-micro-092412-155749 (2015).

63. Almeida, A. et al. A new genomic blueprint of the human gut microbiota. Nature https://doi.org/10.1038/s41586-019-0965-1 (2019)

64. Liu, X., Ser, Z. \& Locasale, J. W. Development and quantitative evaluation of a high-resolution metabolomics technology. Anal. Chem. https://doi.org/ $10.1021 / \mathrm{ac} 403845 \mathrm{u}(2014)$.

65. Pfaffl, M. W. A new mathematical model for relative quantification in realtime RT-PCR. Nucleic Acids Res. https://doi.org/10.1093/nar/29.9.e45 (2001).

\section{Acknowledgements}

We thank Vincent Galy for protocol to develop anti-LGG-1 antibodies, Malene Hansen for LGG-1 reporter strain, Dr. Sidney Kushner for generous help developing HB101
RNAi-competent derivative, and Xiaojing Liu and Juan Liu for optimizing and running fluorometabolite LC-MS analyses. We are very grateful to Filipe Cabreiro for sharing LC-MS protocol, E. coli strains, and general advice. We thank Bob Nakamoto and Yelena Peskova for training and use of their French Press. We thank Chenyu Yang, Noel Higgason, Alexandra Loperfito, Ahtesham Najeeb Chaudhry, and Meghna Shankar for help quantitating fertility images, and specially Nella Solodukhina, Leila Rayyan, and Mikayla Marraccini for help preparing reagents and conducting some of the experiments. We are grateful to the Keck Center for Cellular Imaging for the usage of the Leica SP5X microscopy system (PI: AP; NIH-RR025616). We acknowledge the C. elegans strains provided by the CGC, which is funded by NIH Office of Research Infrastructure Programs (P40 OD010440). Some E. coli strains were provided by CGSC, which is funded by NSF/Biological Infrastructure/Living Collections Program (DBI-0742708). The HSP60 and 4A1(Tubulin) antibodies were obtained from the Developmental Studies Hybridoma Bank, created by the NICHD of the NIH and maintained at The University of Iowa Department of Biology. We thank Kevin Janes and Ariel Pani for revising this manuscript. We thank UVA DoubleHoo, College Council Minerva Research Grant, Ingrassia Family Research Award, and the Jefferson Foundation for support of trainees involved in this work. This work would not have been possible without the generous support of the W. M. Keck Foundation and PEW Charitable Trust.

\section{Author contributions}

E.J.O'R. conceived the study. W.K., J.A.S., M.H., A.D.-W., V.K.M., S.B., and E.J.O'R performed experiments in C. elegans. C.Y., S.Z., G.P., and J.L. performed LC-MS of E. coli and C. elegans samples. C.J. designed and built screening libraries. J.A.S., M.H., V.K.M., and E.J.O'R. conducted Keio and RNAi screens. W.K. and J.A.S. performed data analysis and statistics. E.J.O'R. and J.A.S. conceived of the study. E.J.O'R., J.A.S., and W.K. wrote the manuscript. G.P., N.E.L., J.L., and E.J.O'R. oversaw the study. W.K. and J.A.S. contribute equally as co-first authors.

\section{Competing interests}

The authors declare no competing interests.

\section{Additional information}

Supplementary information is available for this paper at https://doi.org/10.1038/s41467 020-16220-w.

Correspondence and requests for materials should be addressed to N.L. or E.J.O'R.

Peer review information Nature Communications thanks Nektarios Tavernarakis and the other, anonymous, reviewers for their contribution to the peer review of this work.

Reprints and permission information is available at http://www.nature.com/reprints

Publisher's note Springer Nature remains neutral with regard to jurisdictional claims in published maps and institutional affiliations.

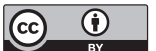

Open Access This article is licensed under a Creative Commons Attribution 4.0 International License, which permits use, sharing, adaptation, distribution and reproduction in any medium or format, as long as you give appropriate credit to the original author(s) and the source, provide a link to the Creative Commons license, and indicate if changes were made. The images or other third party material in this article are included in the article's Creative Commons license, unless indicated otherwise in a credit line to the material. If material is not included in the article's Creative Commons license and your intended use is not permitted by statutory regulation or exceeds the permitted use, you will need to obtain permission directly from the copyright holder. To view a copy of this license, visit http://creativecommons.org/ licenses/by/4.0/.

(C) The Author(s) 2020 